\title{
KABOOM: An Agent-Based Model for Simulating Cognitive Style in Team Problem Solving
}

\author{
Samuel Lapp ${ }^{1}$, Kathryn Jablokow ${ }^{2}$, and Christopher McComb ${ }^{1}$ \\ ${ }^{1}$ College of Engineering, The Pennsylvania State University, University Park, PA, USA \\ ${ }^{2}$ Penn State Great Valley, Malvern, PA, USA
}

\begin{abstract}
The performance of a design team is influenced by each team member's unique cognitive style - i.e., their preferred manner of managing structure as they solve problems, make decisions, and seek to bring about change. Cognitive style plays an important role in how teams of engineers design and collaborate, but the interactions of cognitive style with team organization and processes have not been well studied. The limitations of smallscale behavioral experiments have led researchers to develop computational models for simulating teamwork; however, none have modeled the effects of individuals' cognitive styles. This paper presents KABOOM (KAI Agent-Based Organizational Optimization Model), the first agent-based model of teamwork to incorporate cognitive style. In $\mathrm{KABOOM}$, heterogeneous agents imitate the diverse problem-solving styles described by Kirton's Adaption-Innovation construct, which places each individual somewhere along the spectrum of cognitive style preference. Using the model, we investigate the interacting effects of a team's communication patterns, specialization, and cognitive style composition on design performance. By simulating cognitive style in the context of team problem solving, KABOOM lays the groundwork for the development of team simulations that reflect humans' diverse problem-solving styles.
\end{abstract}

Corresponding author Christopher McComb mccomb@psu.edu

Published by Cambridge University Press under the Creative Commons Attribution licence (http://creativecommons.org/ licenses/by/3.0/)

(C) The Author(s)

Des. Sci.

journals.cambridge.org/dsj DOI

Design Society

a worldwide community

\section{Introduction}

Current design research frequently draws conclusions based on small scale behavioral experiments. Though valuable, these studies are severely limited in scope, and the results are difficult to generalize. The future of design cognition analysis will increasingly need to leverage computational methods for modeling and analyzing both individual and team behavior to enable larger scale studies. In addition, design cognition research must continue its trend toward more rigorous modeling of individual cognitive differences among designers to support more accurate simulations; cognitive style, or one's preference for managing structure in solving problems, is one key example. In general, cognitive style describes patterns in problem-solving behavior and social interactions that result from an individual's unique cognitive processes. For example, one measure of cognitive style differentiates verbal and visual learners [Riding, 1997], while another distinguishes more adaptive and more innovative thinkers [Kirton, 2003]. Cognitive style varies widely across engineers and designers, and differences in cognitive style can have positive or negative effects on the problem-solving performance of an individual or team [Kirton, 2003, Jablokow, 2008, Jablokow et al., 2015a, Sonalkar et al., 2017]. While researchers are beginning to develop simulation approaches for studying team problem solving, current methods do 
not yet attempt to model the influence of cognitive style on individual and team activities. To address this gap, we present in this paper an agent-based model for assessing how individuals' cognitive styles impact an engineering team's problem-solving performance, thereby linking cognition, behavior, and design activity.

Cognitive style is assessed using psychometric instruments such as the Kirton Adaption-Innovation (KAI) inventory [Kirton, 1976]. While there are many ways to measure cognitive differences between people, KAI has been broadly studied and shown to have wide-reaching effects on problem solving behavior [Kirton, 2003, 1976, Sonalkar et al., 2017, Jablokow and Booth, 2006, Jablokow et al., 2015b,a]. Therefore, although it is only one construct for explaining cognitive differences, KAI offers great potential for this vein of research. The KAI inventory measures an individual's relative preference for structure along a bipolar continuum between two equally valued extremes (highly adaptive and highly innovative, respectively). More adaptive individuals prefer more structure in their problem solving (with more of it consensually agreed) and therefore tend to make incremental improvements within the current system to improve and enhance it. In contrast, more innovative individuals prefer less structure, with less concern about consensus, and tend to make radical changes that may ignore rules in an attempt to make the current system work "differently"; their efforts may or may not lead to improvements in that system. Kirton's A-I cognitive style influences both individual problem-solving characteristics and team interactions, and its effects have been studied in observations of engineering teams [Sonalkar et al., 2017, Jablokow and Booth, 2006, Jablokow et al., 2015b,a]. However, it is unclear how cognitive style as measured by KAI could be used to inform the formation of engineering teams.

In fast moving projects, team formation is a critical task that managers must perform using their best guesses about optimal team composition and structure [Levitt, 2012]. As engineering teams become more interdisciplinary and develop increasingly complex structures, selecting the right members for a team is becoming more difficult as well [Crowder et al., 2012]. However, research on optimal team formation strategies is limited. Research on collaboration in team performance is often based on qualitative descriptions and small studies [Bergner et al., 2016]. In vivo studies of design teams over long periods of time are expensive, and the results are often applicable only in a specific context [Perisic et al., 2016].

In light of these issues, agent-based models of team problem solving can serve as a powerful tool for design cognition analysis, because they can quickly compare many different team compositions, structures, and processes [Crowder et al., 2012]. Also, simulation can be useful for isolating independent variables in studies of cognition or social interaction [Singh et al., 2013, e.g.], which is difficult in a human subjects study. Current methods in design cognition analysis can simulate team problem solving involving agent communication [Fan and Yen, 2004, Perisic et al., 2016, e.g.], sometimes with learning mechanisms [Hulse et al., 2017, McComb et al., 2015, e.g.] and social interaction [Tsvetovat and Carley, 2004, Singh et al., 2013], and for some real-world design problems [Zurita et al., 2017, Levitt, 2012, e.g.]. However, none of these methods address the role of cognitive style in problem solving and team performance explicitly.

This paper presents the KAI Agent-Based Organizational Optimization 
Model (KABOOM), the first agent-based modeling framework for studying the problem-solving performance of teams of individuals with diverse cognitive styles. In KABOOM, individual agents exploring a solution space in order to maximize an objective are used to model human problem solving. Each agent has a unique cognitive style to reflect the range of styles measured by the Kirton Adaption-Innovation inventory (KAI). An agent's cognitive style influences its exploration and evaluation of the solution space. The interaction and collaboration between agents also depends on the agents' respective cognitive styles. The goals of this research are: (1) to construct an agent-based model with agents that reflect the diverse cognitive styles of humans across the Adaption-Innovation spectrum; (2) using the model, analyze the performance of individual agents and teams solving a design problem; and (3) investigate how cognitive style impacts the effects of team specialization and communication on performance. As with any model, the assumptions made here limit the extent to which the model reflects real-world scenarios. Rather than predicting a specific team's performance on a real-world problem, the end goal of this model is to investigate and understand the relationships between cognitive style and various aspects of team process (such as communication) and structure (such as specialization). Two outcomes of this research are: (1) hypotheses about cognitive style that can be investigated in human subjects studies; and (2) heuristics related to cognitive style and team work that can support effective team management. After reviewing related work in Adaption-Innovation theory and agent-based modeling, this paper describes the development of KABOOM and discusses the results of several computational experiments on team specialization, communication, and cognitive style composition.

\section{Background}

\section{The Kirton Adaption-Innovation inventory}

The Kirton Adaption-Innovation inventory (KAI) is a psychometric instrument designed to measure a person's cognitive style on a continuous spectrum that ranges from "highly adaptive" to "highly innovative" [Kirton, 2003]. In general terms, more adaptive problem solvers aim to do things better by using incremental changes to continuously improve a system or solution. In contrast, more innovative problem solvers prefer to do things differently, pursuing radically different solutions with more regard for originality than quality. Innovators tend to challenge the existing structures and constraints of a problem, while adaptors tend to support and stay within preexisting structures and bounds.

No cognitive style is universally better than another. However, one cognitive style is sometimes advantageous over others for a specific design problem. For example, problems that require adherence to a given structure, meticulous attention to detail, and conformance to specific rules or standards (e.g., repairing an antique grandfather clock, tuning a nuclear reactor) will tend to favor a more adaptive approach, although more innovative methods will still yield some kind of solution. In contrast, problems that require spanning multiple disciplines, taking a systems view, and challenging current practice (e.g., creating a disruptive product, "breaking" a patent) will tend to favor a more innovative approach, although more adaptive methods will still lead to some kind of progress.

The KAI has 32 items, each answered on a 5-point scale [Kirton, 1976]. 
In addition to an overall KAI score, the instrument provides three sub-scores: Sufficiency of Originality (SO), Efficiency (E), and Rule/Group Conformity (RG) [Kirton, 1976] that are related to different aspects of cognitive style. The Sufficiency of Originality sub-score relates to the quantity and paradigmrelatedness of the solutions a person generates. More adaptive individuals tend to generate fewer ideas (based on cognitive preference, not ability); these ideas tend to be paradigm-preserving and are easier to integrate into existing systems. More innovative individuals tend to generate a higher number of solutions (again, based on preference, not capacity); these ideas tend to be more paradigm-breaking and can be difficult or even harmful to integrate into the existing system [Kirton, 1976]. The Efficiency sub-score describes an individual's attention to detail and methodological approach. More adaptive individuals prefer incremental changes to a solution that are sure to improve quality. In contrast, more innovative individuals may alter a solution in riskier, less well-defined ways, with less regard for the resulting quality [Kirton, 1976]. The Rule/Group Conformity subscore describes an individual's preference for adhering to constraints and norms, as well as their cohesion with a group (i.e., social structure). More adaptive individuals prefer to leverage the prevailing rules, guidelines, and norms in their problem solving; they also tend to promote group cohesion and continuity. More innovative individuals, however, are more likely to disregard the prevailing rules and norms, and are less concerned about conforming to a group; they may actively move away from group consensus and can cause disruption or discord in a team [Kirton, 1976]. The methods section describes how each of these subfactors of KAI inform the development of agents that have their own cognitive styles.

\section{Cognitive style diversity and design teams}

When individuals work together, their diverse cognitive characteristics can influence their collaborations in both positive and negative ways. Kirton uses the term cognitive gap to describe differences in cognitive level (intelligence, experience, knowledge, etc.) and/or cognitive style that can appear between two individuals, an individual and a group, two groups, or between an individual/group and the problem at hand [Kirton, 2003, Jablokow and Booth, 2006]. In previous work related to KAI and teams, Kurtzberg Kurtzberg [2005] studied the creative fluency of homogeneous teams and heterogeneous teams (as categorized by KAI cognitive gaps) and found that heterogeneous teams outperformed homogeneous teams in terms of creative output (i.e., number of ideas). Hammerschmidt [1996] studied team success and cognitive style diversity using KAI and found that teams had higher levels of success when tasks were coordinated with KAI (e.g., a more adaptive task aligned with a more adaptive sub-team). His work also revealed that when sub-teams had similar KAI scores (i.e., were homogeneous), overall team success increased as a result of enhanced inter-team communication, while diverse (i.e., heterogeneous) teams were more likely to fail as a result of unresolved cognitive gaps. Jablokow et al. [2015a] explored the effects of cognitive gaps on dyad performance and interactions between design team members during concept generation. Their results suggest that as the cognitive gap between team members increases, the more adaptive team member tends to feel that they contributed less to team ideation, while the more innovative team 
member tends to feel that they contributed more. In other previous work by Jablokow et al. [2015a], the results suggest that the presence of more innovative individuals on a team may be correlated with a greater occurrence of unique ideas in the team, where "unique" was defined as "new to the current team discussion". They also found that more innovative teams (determined by KAI mean) tended to exhibit team interactions with a higher degree of integration between topics.

\section{Mechanisms of agent-based modeling for design teams}

An Agent-Based Model (sometimes called a Multi-Agent System) is a software simulation method in which autonomous, heterogeneous agents interact with their environment and other agents [Garcia, 2005]. Each agent acts with a set of behavioral rules in order to accomplish an objective [Bonabeau, 2002]. Rather than defining the macroscopic behavior of a system, an agent-based model only defines behavioral rules for individual agents and a structure for agent interactions [Bonabeau, 2002, Jennings, 2000]. Agents make run-time decisions based on limited knowledge of the environment and limited decision-making capabilities [Jennings, 2000, Tsvetovat and Carley, 2004]. In addition to these cognitive constraints, agents may be socially constrained with limited ability to connect and share information with other agents [Tsvetovat and Carley, 2004]. By simulating agent interactions, an agent-based model can capture emergent phenomena that are more than the sum of the individual agents' actions [Bonabeau, 2002].

Some studies investigate the frequency of communication among agents throughout the course of the problem-solving period. In the context of design research, communication refers to the exchange of design solutions [Singh et al., 2013]. A solution is the outcome of a design effort, which, when communicated to a teammate, may prove either beneficial or detrimental in the form of false leads, failures, or flawed concepts. Research on communication in design teams shows that more communication is not necessarily better; there is a trade-off between communication frequency and individual work [Patrashkova-Volzdoska et al., 2003, Patrashkova and McComb, 2004]. Some researchers have shown that intermittent communication can provide the benefits of constant communication, as well as the benefits of individual work [Bernstein et al., 2018]. There are even some cases where no communication is the best team process strategy [McComb et al., 2017a, McComb and Maier, 2018].

Engineering teams often divide the design of complex systems into multiple components that are then addressed or solved by specialized team members with corresponding expertise in a specific domain [Hulse et al., 2017, 2018]. This multi-component approach to complex systems design is analogous to coordination between multiple agents in a computational model: decisions are made with respect to separate components and later aggregated to form a global solution [Zurita et al., 2017]. An agent-based model can reflect the way engineering teams solve complex systems in a distributed manner by assigning agents to specialized sub-teams that each control a subset of all design variables for a global objective function [Hulse et al., 2018]. This approach makes it possible to use an agent-based model to rapidly investigate the effects of team structure on a design team's performance.

Appropriate team composition is critical for high team performance [MartínezMiranda and Pavón, 2012] and should consider not only the personal traits of 
individuals, but also the nature of the problem being addressed by the team [Zhang et al., 2009]. Team composition can have a detrimental effect on performance and social interactions if teams are not carefully constructed and managed [Martínez-Miranda and Pavón, 2012]. Although this paper examines team composition in terms of the cognitive styles of agents on a team, other factors of team composition exist and have been studied both in human subjects studies and through computational modeling. According to Singh et al. [2013], a team's composition refers to its size, life-span (one project or several), location (collocated or geographically distributed), structure (flat or hierarchical), and heterogeneity (homogeneous or heterogeneous). Not surprisingly, a team's composition in terms of domain of expertise also plays a key part in product development success [Brown and Eisenhardt, 1995]. However, there is little evidence regarding how other personal cognitive qualities like cognitive style influence a team's success. Martínez-Miranda and Pavón [2012] state that although some human resource departments use tests of personality and cognitive level:

It could be even more useful for project managers to apply the results of cognitive and psychological tests to build virtual teams and simulate their possible behaviors in order to analyze what could happen when people with specific characteristics interact with each other and with their respective tasks over the entire duration of a project. [Martinez-Miranda and Pavón, 2012]

Among the large number of psychological instruments used today, many have limited validation or scientific support. The Kirton Adaption-Innovation inventory (KAI), however, has been extensively validated and is regularly used for team management [Kirton, 2003]. This research, therefore, aims to fill a gap at the intersection of cognitive style and agent-based modeling by incorporating Kirton's Adaption-Innovation cognitive style characteristics into an agent-based model of engineering teamwork.

\section{State of the art}

In the 1990s, managers of the semiconductor manufacturer "Micro" were faced with the challenge of developing high-performance teams for complex and concurrent projects on an extremely rapid product life cycle [Levitt, 2012]. While the performance of their semiconductor chips could be modeled with computer simulations, managers still used guess-and-check methods for the design of their teams-but they began to ask for computational tools that could simulate team performance in the same way that software simulations could predict the performance of an engineering design [Levitt, 2012]. This led to the Virtual Design Team (VDT) project [Kunz et al., 1998, Levitt, 2012], an early effort to create simulation tools that helped managers design software teams by simulating the assignment and completion of work items. The VDT simulation divides a problem into tasks and types of work, and then uses stochastic discrete-event simulation to model the time and number of errors incurred in completing each task. VDT models real and complex projects, and includes both errors in task completion and noise in communication [Levitt, 2012].

Since then, many researchers have developed models with the aim of reflecting some aspect of human behavior or teamwork [Fernandes et al., 2017, 
Vermillion and Malak, 2015, 2018, e.g.] more accurately. These models vary in their purpose. Some, like VDT, are team management simulation tools that model team performance to avoid the associated costs of long and expensive human subjects studies [Perisic et al., 2016, e.g.]. Other models focus on studying the social aspects of communication and collaboration [Singh et al., 2013, Tsvetovat and Carley, 2004, e.g.]. For example, Tsvetovat and Carley [2004] develops a detailed model of complex social and technological systems by including learning, social network theory, and social psychology in an agent-based model. Recent research in systems engineering by Vermillion and Malak uses agentbased approaches to investigate the delegation of authority and use of incentives in design teams [Vermillion and Malak, 2015, 2018]. Fan and Yen [2004] presents an extensive review of agent-based models addressing communication and collaboration; some of the models reviewed include emotion and sentiment [Fan and Yen, 2004]. However, the authors are not aware of any models that incorporate individual cognitive style for heterogeneous agents.

Recently, several agent-based models have attempted to focus on the personalities, attitudes, and emotions of problem solvers, as well as their knowledge [Martinez-Miranda et al., 2006, Zhang et al., 2009, Dehkordi et al., 2012, e.g.]. For example, the TEAKS framework [Martinez-Miranda et al., 2006, Martínez-Miranda and Pavón, 2012] models the social and emotional aspects of team problem solving. Agent interactions are based on the PECS (physics, emotion, cognition, and social status) framework [Urban and Schmidt, 2001], and personality is described by drive, influence, steadiness, and compliance. Dehkordi et al. [2012] studies work-overload impact by modeling the effects of stress and motivation on team performance, and Zhang et al. [2009] presents a model where human behavior, development process, and organizational structure all influence design outcomes. These models focus on emotions and sentiments to elicit human-like behaviors in computational agents.

Finally, some models implement contextualized, real-world design problems that are solved by teams of agents in an agent-based model [Zurita et al., 2017, McComb et al., 2015, e.g.]. Work by Zurita et al. [2017] is an example of an agent-based model specifically created for a contextualized problem: designing a Formula SAE racing vehicle. Their model demonstrates that by using a cooperative evolutionary algorithm, an agent-based model can design a complex system. Like Hulse et al. [2018], members of the design team adopt specializations by breaking the problem into separate functional subsystems (e.g., engine, suspension, and brakes).

The CISAT framework [McComb et al., 2015, 2017b,a] is another agentbased model that uses contextualized problems to study how problem characteristics affect the optimal team process and team characteristics. The CISAT framework reflects eight characteristics of both team activity and individual cognition, namely: organic interaction timing, quality-informed solution sharing, quality bias reduction, self-bias, operational learning, breadth versus depth solution search, and satisficing [McComb et al., 2015]. In McComb et al. [2017a], the CISAT model is used to find optimal team characteristics based on properties of the problem being addressed. These two models provide much of the inspiration for KABOOM. 


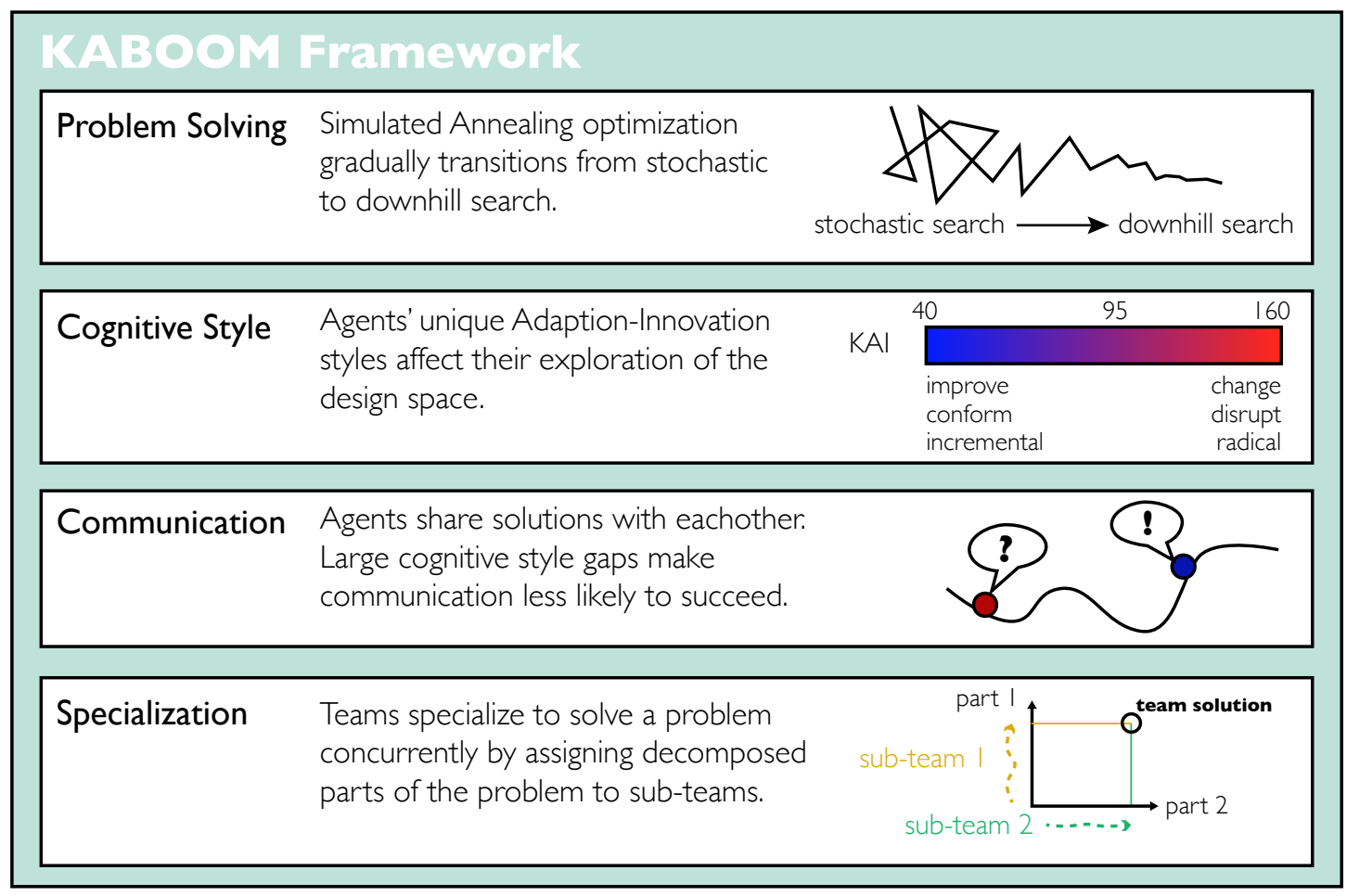

Figure 1. Outline of the KABOOM framework and its key features

\section{Methods}

$\mathrm{KABOOM}$ is the first agent-based model to incorporate cognitive style of heterogeneous agents as a strategy to simulate the problem-solving behaviors of human teams. This section describes: (1) the agent-based model framework for KABOOM; (2) the implementation of Kirton's Adaption-Innovation cognitive style for agents; (3) communication in the model through pairwise solution sharing and team meetings; and (4) creating a virtual population with KAI cognitive style traits. Figure 1 provides a high-level summary of the key components of the model.

\section{The KABOOM framework}

The KAI Agent-Based Organizational Optimization Model (KABOOM) is a multi-agent optimization scheme in which independent software agents explore a solution space by varying parameters and evaluating a cost function. KABOOM is based on a simulated-annealing [Kirkpatrick et al., 1983] optimization algorithm: the agents start with a high "temperature", allowing them to explore widespread solutions non-greedily; the temperature gradually cools over the course of the simulation, leading to a local and more greedy search. The gradual transition from stochastic to downhill search in simulating annealing reflects the nature of human problem solving [Cagan and Kotovsky, 1997, Yu et al., 2016] and has been used in other human problem-solving simulations [McComb et al., 2015, e.g.]. Figure 2 illustrates an agent problem solving in a simulated annealing 
Objective Function

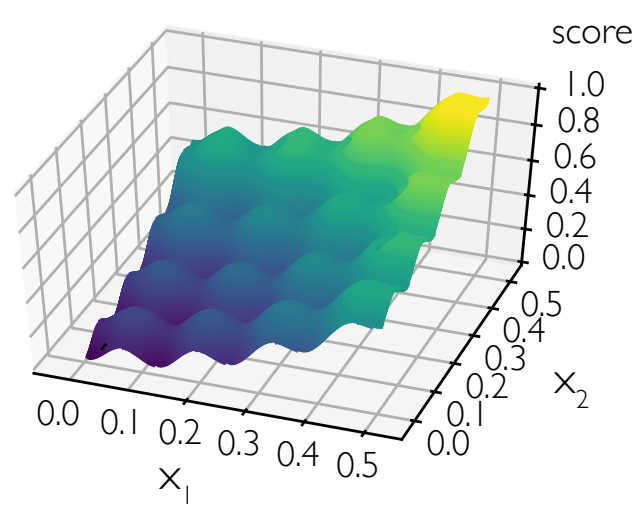

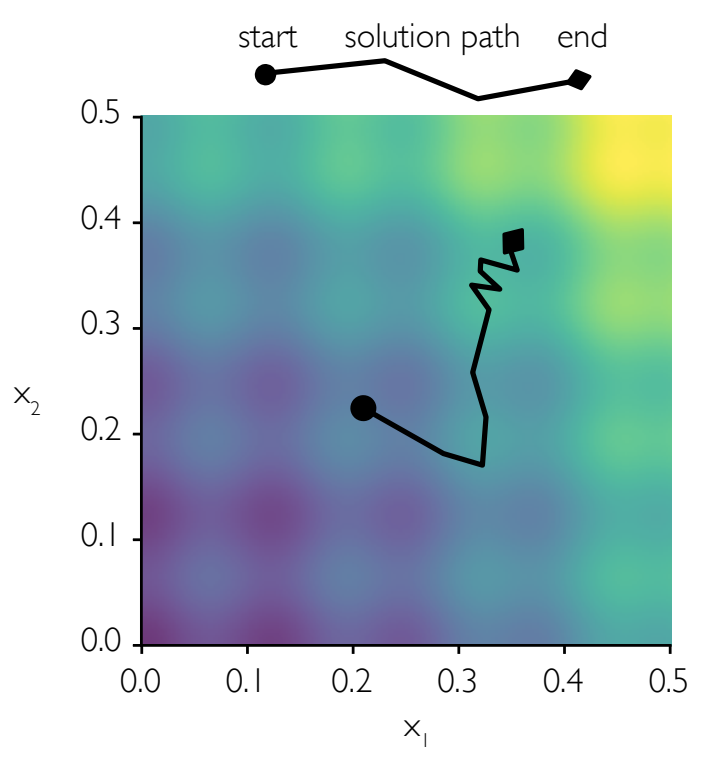

Figure 2. Simulated annealing involves agents exploring a solution space (right) in order to maximize a defined objective function (left). The path on the right shows a series of solutions starting at the circle and ending at the diamond.

framework. The agent explores a 2-dimensional problem space with the goal of maximizing an objective function (left). The black line connects the sequence of solutions explored by the agent over the course of 100 iterations of the simulation, ending at the black diamond. The agent makes large changes to the solution early on, followed by smaller steps towards the end, in accordance with the simulated annealing scheme.

In this paper, a solution is a set of parameters that define a position in the solution space, and the quality of a solution is the value of the objective function for those parameters. Team performance is taken to be the best solution any individual on a team has found. While this approach is the norm in the optimization and modeling literature, future work could consider more subtle measures of performance that include social integrity of the team, selfefficacy, consumption of resources such as money and time, or other measures of performance.

Many computational models of team problem solving assume that agents can perfectly evaluate the objective function for solutions they create. This is due in part to agent-based models being grounded in the optimization literature. In contrast, KABOOM assumes agents can evaluate the quality of their solutions using the objective function, but their perception of solution quality is affected by their respective cognitive styles. Each agent has an assigned cognitive style, which includes the three sub-score dimensions of Sufficiency of Originality (SO), Efficiency (E), and Rule/Group Conformity (RG), in addition to the total score (KAI). These parameters influence how the agent perceives solution quality and how it explores the solution space. The following sections describe how the total KAI score and each sub-score impact agent behavior. 


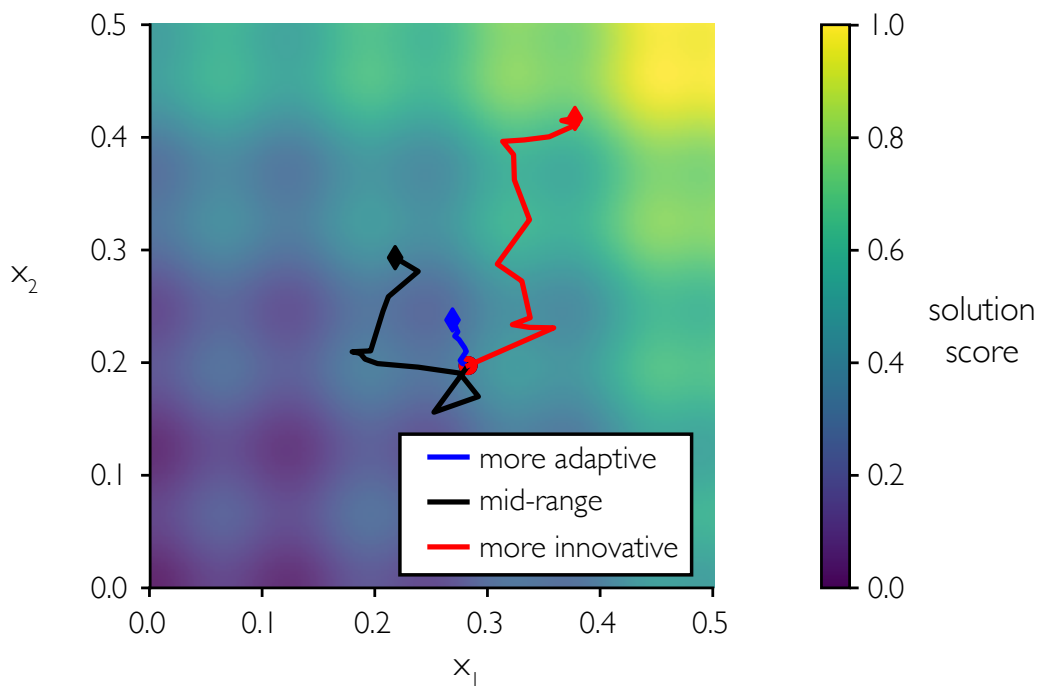

Figure 3. Agents with more adaptive styles (lower KAI scores) move in smaller steps on each iteration, while agents with more innovative styles (higher KAI scores) take larger steps in the solution space.

\section{Simulating cognitive style}

The agents' exploration of the solution space depends on their total KAI score, as well as the three sub-factor scores of Sufficiency of Originality (SO), Efficiency (E), and Rule/Group Conformity (RG). People with higher KAI scores tend to make larger changes to a design in search of a different solution, while people with lower KAI scores tend to make smaller changes to refine an existing solution. In the simulation, the distance an agent moves in the solution space on one turn (iteration) is positively correlated with the total KAI score. Figure 3 illustrates the differences in step size for agents of adaptive, mid-range, and innovative styles in a 2-dimensional problem. When searching for new solutions, a more adaptive agent moves in smaller, incremental steps, tweaking the solution with marginal adjustments. In contrast, a more innovative agent moves in larger leaps, often generating ideas that are distant and very distinct from their current solution.

Each agent has an individual speed parameter with an initial value dependent on their total KAI score. Speed determines how closely or distantly related an agent's subsequent solutions are. Speed is roughly analogous to novelty of solutions in real-world problems, in that high speed will lead to a series of very distinct solutions, while low speed will lead to a series of very similar solutions. The speed decays geometrically throughout the course of the simulation. The distance, $D$, from the current solution to the next solution an agent generates is drawn from a chi $(\chi)$ distribution and scaled by the agent's current speed, $s$ :

$$
D=s \cdot \chi
$$

where $\chi$ is a random variable characterized by the chi probability density function with $k=1.9$ degrees of freedom. The minimum travel distance in one step is set to 
one ten-thousandth of the entire space, and there is no upper bound. The agent's start speed, $s_{0}$, is calculated as

$$
s_{0}=\mu_{s}+K A I^{*} \cdot \sigma_{s}
$$

where $K A I^{*}$ is the standardized KAI score (re-scaled for a mean of 0 and a standard deviation of 1), $\mu_{s}$ is the average starting speed for all agents, and $\sigma_{s}$ is the standard deviation of starting speed across all agents.

\section{Model parameters}

Parameters in the model are generally tuned to reflect observed human behavior. Except where noted, the parameters were held constant at the values listed in Table 1. An appendix contains a table listing all parameter values for each figure in the paper.

Table 1. List of model parameters and their default values

\begin{tabular}{lccl} 
Parameter & Symbol & Value & Description \\
\hline General Parameters & & & \\
\hline Iterations & & 300 & number of simulation steps in one simulation \\
Team size & & 6 & number of agents on the team \\
Number of sub-teams & & 2 & number of specialized teams \\
Agents per sub-team & & 3 & number of agents on each sub-team \\
Average starting temperature & $\mu_{T}$ & 1 & \\
Standard deviation of temperature & $\sigma_{T}$ & 0.8 & \\
Average starting speed & $\mu_{s}$ & 0.01 & \\
Standard deviation of speed & $\sigma_{s}$ & 0.007 & \\
& & & \\
Objective Function Parameters & & & \\
\hline Oscillation amplitude & $\alpha$ & 0.1 & \\
Scaling parameter & $\beta$ & 1 & \\
Feasible solution space & & {$[-1,1]$} & each dimension is bounded by this range \\
& & & \\
Communication Parameters & & & \\
\hline Communication frequency & $c$ & 0.2 & probability an agent communicates on turn \\
Meeting interval & & 50 & number of iterations between team meetings
\end{tabular}

\section{Efficiency}

Efficiency describes an individual's preference for structure in their working methods, which range from applying highly detailed and incremental improvements to a solution (more adaptive) to dramatically or tangentially altering a solution, with less regard for detail and quality (more innovative). The temperature parameter in simulated annealing determines the probability of an agent accepting a candidate solution that does not improve the objective function relative to their own current solution. In a real-world design setting, temperature 
corresponds to exploration. Early on in the design process, designers explore new solutions with little regard for quality in order to expand the known solution space. As the design process continues, solution quality becomes more important and exploration transitions to exploitation.

In KABOOM, an agent's starting temperature is correlated with the E subscore. Temperature always decreases geometrically over the course of the simulation, but both the start temperature $T_{0}$ and the cooling rate are a function of E. An agent with a higher (more innovative) E sub-score starts with a higher temperature, and thus a higher probability of accepting solutions that do not improve the objective function. This can cause them to leave good solutions behind and miss high-quality nearby solutions, but it can also allow them to escape local minima to find better solutions in a "rough" optimization topology. Agents with higher Efficiency scores also cool down slower, meaning they might never reach a very low temperature or refine a single local solution. In contrast, an agent with a lower (more adaptive) E sub-score starts with a lower temperature, and thus a lower probability of accepting solutions that do not improve the objective function. This can cause them to quickly achieve locally optimal results, but it can also lead them to become stuck in local minima. The resulting behavior of the agents overall is that the more adaptive agents choose a solution early on and polish it to perfection, while the more innovative agents spend most of their time exploring very diverse solutions without refining them. The agent's start temperature $T_{0}$ is calculated as

$$
T_{0}=\mu_{T}+E^{*} \cdot \sigma_{T}
$$

where $E^{*}$ is the standardized Efficiency sub-score (re-scaled for a mean of 0 and a standard deviation of 1$), \mu_{T}$ is the average temperature for all agents, and $\sigma_{T}$ is the standard deviation of temperature across all agents.

The agent's ratio of start temperature $r_{0}$ to final temperature $r_{f}$ is

$$
\frac{r_{0}}{r_{f}}=\frac{1}{\exp \left(2-E^{*}\right)}
$$

which is bounded by $10^{-10}$ and 1 . The geometric decay ratio for each time step is calculated based on start temperature, start-to-end ratio, and the number of steps in the simulation. Figure 4A shows the temperature over the course of a simulation for agents with more innovative (high E), mid-range (mid-range E), and more adaptive (low E) Efficiency sub-scores. The agent's speed decays with the same ratio as the temperature.

In simulated annealing, the probability that an agent will accept or reject a solution that is inferior to its current solution depends on its temperature and the difference in solution quality according to Equation 5 [Kirkpatrick et al., 1983]. At high temperatures, an agent will accept solutions regardless of quality, but at low temperatures, agents only accept solutions that improve the objective function, as shown below:

$$
P_{\text {accept }}=\exp \left(\frac{f\left(\vec{x}_{n}\right)-f(\vec{x})}{k_{B} T}\right), \quad f\left(\vec{x}_{n}\right)<f(\vec{x})
$$

where $P_{\text {accept }}$ is the probability that an agent with current solution $\vec{x}$ accepts a 


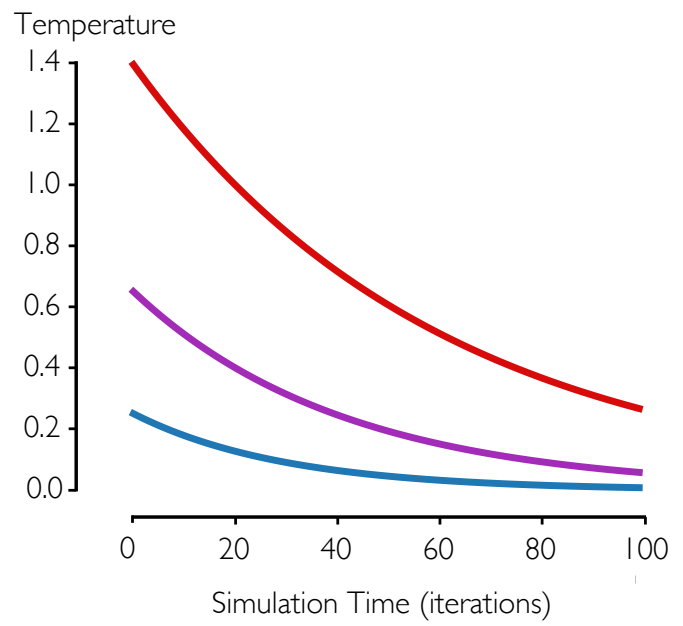

(A)

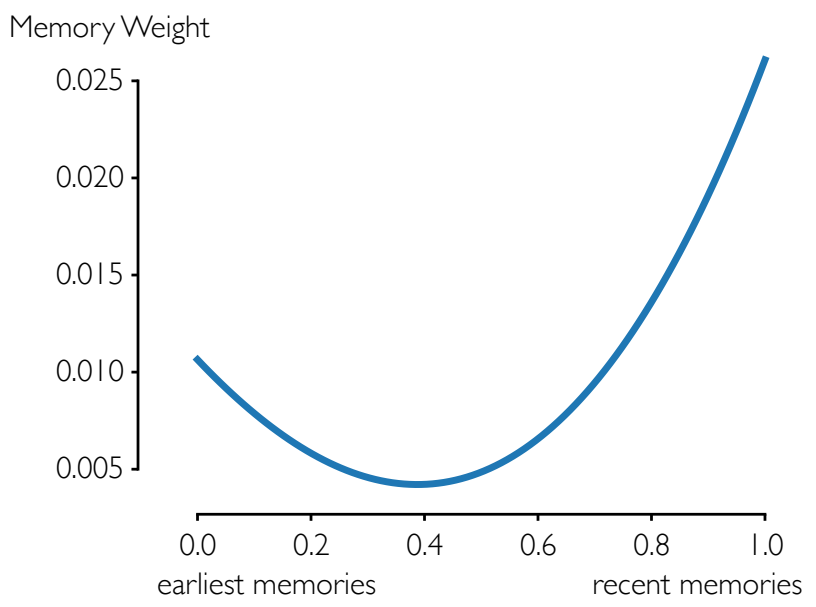

time of memory

(B)

Figure 4. (A) Cooling schedules for agents with different Efficiency sub-scores (B) In calculating the perceived memory location, an agent's past memories are weighted based on the serial position effect reflected in this curve: the most recent memories and earliest memories are recalled more easily than intermediate memories.

new solution $\vec{x}_{n}$ that does not improve the objective function $f$ as a function of current temperature $T$ and a constant $k_{B}$. We can absorb $k_{B}$ into the calculation of temperature.

Figure 5 illustrates the effect of the Efficiency style sub-factor on three agents' exploration of the solution space. Compared to the mid-range agent (black), the more adaptive agent (blue) refines an early solution, while the more innovative agent (red) continuously explores without refining a solution.

\section{Sufficiency of originality}

An agent's desire to retain and modify known solutions or, conversely, to explore more unfamiliar ideas, is related to the Sufficiency of Originality sub-score of KAI. When agents evaluate a candidate solution in KABOOM, more adaptive agents are more likely to accept a candidate solution that directs them towards their previous solutions and are less likely to accept a solution that leads them away from previous solutions. On the other hand, more innovative agents prefer to choose ideas that move them further from ideas they have explored in the past and to reject ideas that lead them towards their previous solutions. An agent's perception of its previous solutions is represented by a single point in the solution space, which is a weighted mean (centroid) of all of their previous positions or "memories". The weights on an agent's memories are strongest for the most recent memories, weakest for middle memories, and intermediate strength for the earliest memories (Figure 4B). This reflects the serial position effect [Colman, 2015], which describes how people tend to remember early memories (primacy effect) and recent memories (recency effect) more readily than intermediate memories.

The direction of the weighted memory position from the current position is a weighted average of the directions to all previous solutions the agent has visited. 


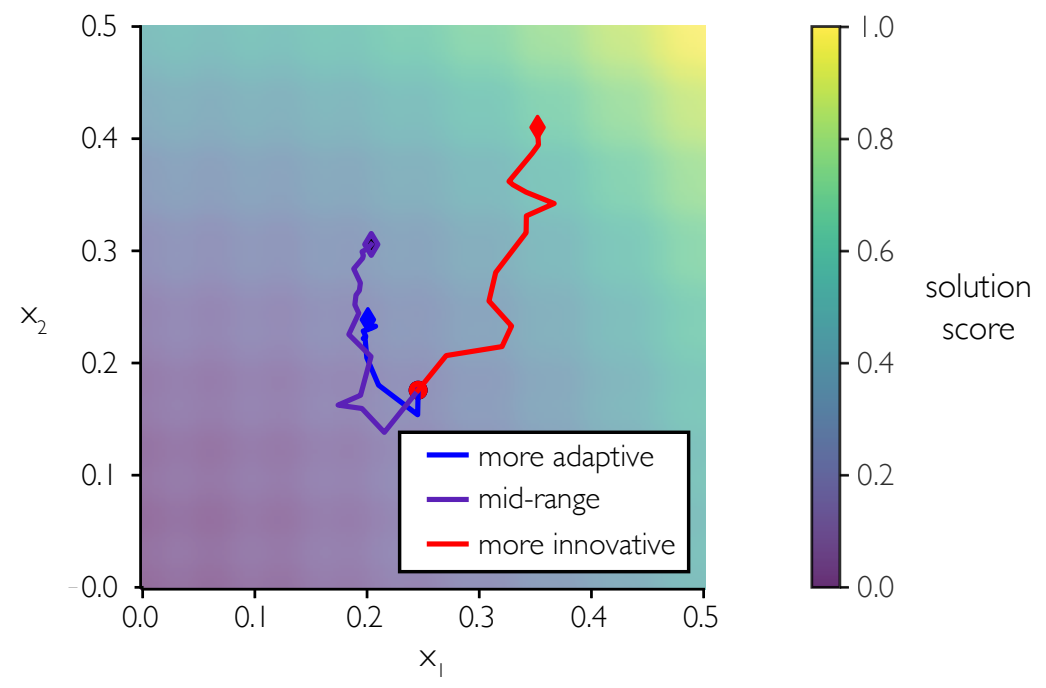

Figure 5. Effect of E sub-factor on solution space exploration

We call $\vec{v}_{m e m}$ the perceived memory direction, where the weights are given by the primacy-recency bias function $Q$ :

$$
\vec{v}_{m e m}=\sum_{n=1}^{N}\left(\vec{x}-M_{n}\right) * Q(n)
$$

and where the memory $\boldsymbol{M}$ is a list of $\mathrm{N}$ previous solutions (each memory being a vector of the same shape as the current solution $\vec{x}$ ). The primacy-recency bias function for memory $\mathrm{n}$ of $\mathrm{N}$ total memories is:

$$
Q(n)=n^{3}+0.4(N-n)^{3}
$$

which gives a U-shaped curve with stronger biases for the earliest and most recent memories, respectively, than for other memories (Figure 4B). The weights are calculated with this equation then normalized so that they sum to 1 .

The direction of the candidate solution from the current solution is the vector difference between the candidate solution and the current solution, $\vec{v}_{n}=\vec{x}_{n}-\vec{x}$. The dot product of these two directions indicates whether the new solution is in the direction of the perceived memory or away from the perceived memory. We call this dot product the originality $\Omega$ :

$$
\Omega=\vec{v}_{m e m} \cdot \vec{v}_{n}
$$

Then, the Sufficiency of Originality preference $P_{S O}$ is:

$$
P_{S O}=\Omega \cdot S O^{*} \cdot W_{S O}
$$

where $S O^{*}$ is the standardized SO score (re-scaled for a mean of 0 and a standard deviation of 1), and $W_{S O}$ is a global scaling constant that determines the strength 


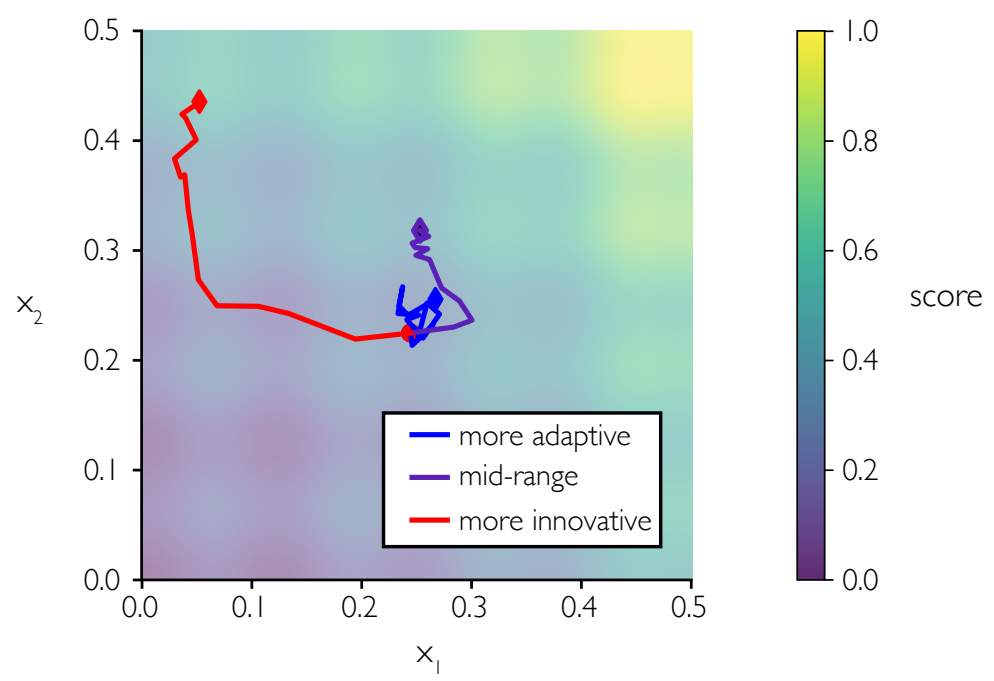

Figure 6. Effect of SO sub-factor on solution space exploration

of the SO preference. For this paper, we set $W_{S O}$ to 2 , which creates a range of behaviors for agents across the KAI cognitive style spectrum. When an agent evaluates the quality of a candidate solution, the SO preference $P_{S O}$ and RG preference $P_{R G}$ (explained below) influence the perceived solution quality: the preferences are added to the true value of the objective function for the candidate solution. In other words, the perceived solution quality $f_{P}$ of a candidate solution $\vec{x}_{n}$ is related to the true solution quality $f\left(\vec{x}_{n}\right)$ by

$$
f_{P}(\vec{x})=f(\vec{x})+P_{S O}+P_{R G}
$$

Figure 6 illustrates the effect of the SO cognitive style sub-factor on three agents' exploration in a 2-dimensional solution space. The black line shows the solution path of an agent with a mid-range cognitive style. The red line shows the path of a more innovative agent in the same space, with only the Sufficiency of Originality preference active (and otherwise identical to the mid-range agent). The innovative SO style gives the agent a preference for solutions that move it away from its previous solutions, leading the agent to explore further into the corner of the solution space. On the other hand, the blue line shows the more adaptive agent's preference for solutions that are close to its previous solutions, leading to a dense packing of solutions near its original starting point.

\section{Rule/group conformity}

The third cognitive style sub-score, Rule/Group Conformity (RG), describes an individual's preference for managing structure in both impersonal and personal contexts. More adaptive individuals prefer to leverage existing impersonal structures, such as rules, guidelines, and precedents, while more innovative individuals are more likely to bend or violate these structures. A similar pattern of behavior emerges for personal structures, such as groups or teams. A person 
with an adaptive Rule/Group Conformity style tends to cohere with the group and seek group consensus, while a more innovative person tends to diverge from and may even disrupt the group.

In order to capture one aspect of the Rule/Group Conformity sub-factor, the present model focuses on conformity to personal structures (i.e. an individual's desire to converge or diverge from group consensus). Future work could develop other aspects of Rule/Group Conformity, for instance, by modifying the agents' adherence to optimization constraints. In KABOOM, the more adaptive agents are more likely to move towards solutions that bring them closer to the team's average position, thus encouraging group cohesion. More innovative agents, on the other hand, have a preference for solutions that move them away from the mean position of the group.

In KABOOM, this aspect of Rule/Group Conformity is implemented in a similar way to Sufficiency of Originality by replacing an agent's memories with the current solutions. In other words, while SO makes agents favor solutions towards (more adaptive) or away from (more innovative) their own previous solutions, RG gives agents a preference towards (more adaptive) or away from (more innovative) the current solutions of their teammates (Figure 7).

A "team position" $\vec{x}_{\text {team }}$ is represented by the centroid of the current solution of each member of the team:

$$
\vec{x}_{\text {team }}=\frac{1}{N} \sum_{n=1}^{N} T_{n}
$$

where $\mathrm{T}$ is the team of $\mathrm{N}$ agents, and $T_{n}$ selects the current solution of each agent on the team. As before, the direction of the candidate solution from the current solution is the vector difference, $\vec{v}_{n}=\vec{x}_{n}-\vec{x}$. Likewise, the direction of the team position from the current position is $\vec{v}_{\text {team }}=\vec{x}_{\text {team }}-\vec{x}$.

The dot product of these two vectors indicates whether the new solution is in the direction of the team (positive dot product) or away from the team (negative dot product). We call this dot product the conformity $C$ :

$$
C=\vec{v}_{\text {team }} \cdot \vec{v}_{n}
$$

Then, the group conformity preference $P_{R G}$ is:

$$
P_{R G}=C \cdot R G^{*} \cdot W_{R G}
$$

where $R G *$ is the standardized Rule/Group Conformity sub-score (re-scaled for a mean of 0 and a standard deviation of 1 ), and $W_{R G}$ is a global constant used to change the strength of the RG preference, which is set to 2 to create a range of behaviors.

Figure 7 illustrates the effect of the Rule/Group Conformity cognitive style sub-factor for adaptive and innovative teams, respectively, of three agents each. Agents with a more adaptive RG style prefer solutions that bring them towards their team, resulting in team convergence (left), while more innovative agents seek solutions away from their team, leading to team divergence (right). 

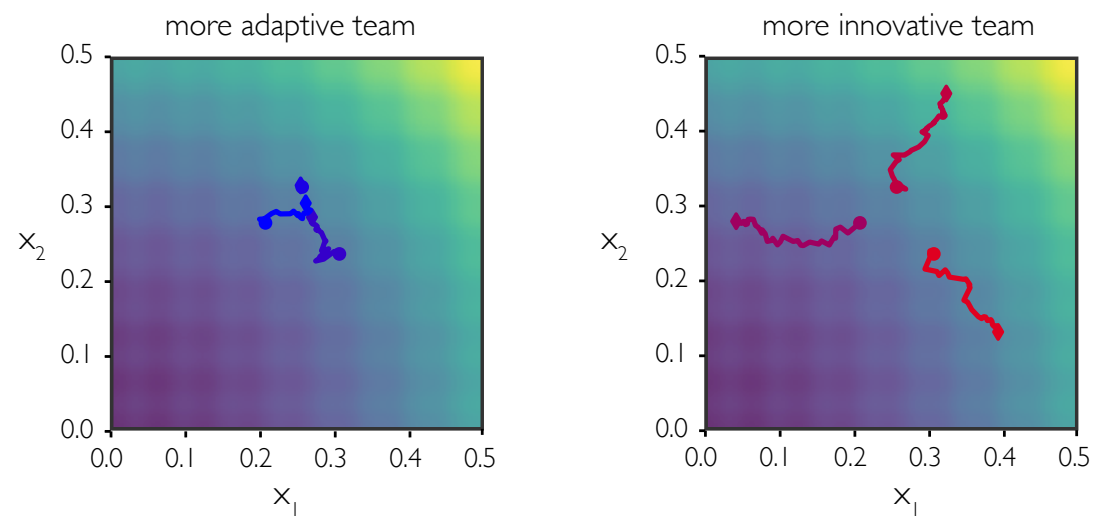

Figure 7. Effect of Rule/Group Conformity sub-factor on homogeneous teams of adaptive style and innovative style, respectively (blue paths show adaptive agents and red paths show innovative agents)

\section{Interactions and communication}

In models of team problem solving, communication can be modeled using organically timed pairwise interactions between team members [McComb et al., 2015]. In KABOOM, agents collaborate and share solutions by sharing their current positions in the solution space with each other. While communication can be influenced by many social and individual factors (as in [PatrashkovaVolzdoska et al., 2003, Singh et al., 2013]), this work focuses specifically on the influence of cognitive style on communication. On a given turn, an agent can choose to either explore a new solution individually or communicate with another agent to share solutions. The probability of an agent choosing to communicate in a pairwise interaction on a given turn is set by a model parameter $c$, which can be constant in time or change over time. On each turn, agents who decide to collaborate are paired randomly, regardless of their sub-team. While future work may give agents preferences for communicating with specific individuals, groups, or networks, the current model chooses the nominal case where any agent interacts with any other without preference. If there is an odd number of agents who wish to collaborate, the unpaired agent explores individually for that turn.

After sharing their current solutions, each agent evaluates the solution shared with them and chooses whether they want to move to that solution. As with evaluating a potential solution in individual exploration, the probability of accepting a solution that does not improve the objective function in favor of the current one is a stochastic function of the agent's temperature (Equation 5). When the temperature is zero, there is zero probability of choosing a solution that does not improve the objective function.

Research shows that when the difference in two people's KAI total scores is greater than 20 points, communicating ideas with each other tends to become increasingly difficult [Kirton, 2003]. Agent collaboration in KABOOM reflects this increasing difficulty in communication due to the cognitive gap in style. In the model, communication between two agents always has some probability of failing; this likelihood is positively correlated with the difference between the agents' KAI total scores (i.e., their cognitive style gap). This is implemented by 
requiring a uniformly distributed random variable to be less than the difference in two agents' KAI total scores for successful communication. If communication is not successful, no information is shared between the agents, essentially resulting in a wasted iteration.

The probability of successful collaboration is:

$$
P= \begin{cases}1, & \text { if } \Delta K A I \leq 10 \\ 1-(\Delta K A I-10) / 170, & \text { if } \Delta K A I>10\end{cases}
$$

where $\triangle K A I$ is the difference between the two agents' total KAI scores. Agents with KAI total score differences of 10 points or less have a $100 \%$ success rate, which drops linearly beyond the 10-point just-noticeable-difference (JND) established in prior research [Kirton, 2003]. Communication across extremely large gaps of 100 points is modeled as only being successful $50 \%$ of the time (the observed KAI range is 109 points [Kirton, 1976]).

\section{Team meetings}

In addition to pair-wise communication, agent teams have regularly scheduled meetings in which all team members converge to a single solution. First, the team creates an aggregate solution from the specialized sub-teams: each sub-team finds the best solution from any of its agents, then contributes only its dimensions to the aggregate team solution. For example, if there is a 2-dimensional solution space where sub-team 1 controls $x_{1}$ and sub-team 2 controls $x_{2}$, the aggregate solution is $\left\langle x_{1}\right.$ from sub-team $1, x_{2}$ from sub-team $2>$. Finally, all agents accept the new aggregate position, regardless of the quality of the new aggregate solution.

\section{Creating a virtual population with KAI}

We create a virtual population of individuals such that the distributions and correlations of KAI total scores (KAI total) and sub-scores (SO, E, and RG) reflect those of the general population. A Python script generates KAI scores and sub-scores for 10,000 virtual individuals from a multivariate normal distribution. The mean, standard deviation, and correlations of KAI total, SO, E, and RG are based on a dataset of 597 individuals' scores and sub-scores gathered in previous research [Jablokow, 2008]. The sub-scores are all imperfectly correlated $\left(0.4<R^{2}<0.8\right)$ with the total KAI score. Because of this, even agents with the same KAI score will have different sub-scores for SO, E, and RG, meaning that any two agents with the same total KAI score are unlikely to be precisely identical in their style (as with humans). KABOOM draws from this virtual population to create agents with associated KAI scores. When the model requests an agent with a specific total KAI score (for instance, 95), it receives a randomly selected member from all individuals in the virtual population having the requested KAI score. Thus, requesting two agents with KAI total scores of 95 will return two different agents, each having total scores of 95 but (likely) having different subscores for SO, E, and RG. 


\section{Selecting agents for a team}

When forming a team of agents, KABOOM can select individuals from the virtual population randomly or according to a team composition rule. This paper uses three team composition strategies:

1. Organic Composition: team members are drawn randomly from a virtual population that is statistically representative of the true distribution of KAI scores;

2. Homogeneous Composition: all team members have the same KAI total score (but likely have different sub-scores);

3. Linearly Distributed Heterogeneous Composition: the team is composed with a given mean and range of KAI total scores. The team will be composed of agents linearly distributed across the range and centered on the mean. For example, a linearly distributed 5-person team with mean KAI of 100 and a range of 40 will be composed of five agents with KAI scores $80,90,100,110$, and 120 .

When teams are divided into specialized sub-teams, each sub-team (rather than the full team) is selected to have a linearly distributed composition with the given mean and range. For example, given a team of 4 with 2 sub-teams of 2, and requiring a KAI mean of 100 and range of 40, each sub-team will be composed of two agents with KAI scores of 80 and 120 .

It is worth noting that, in real management scenarios, managers often must compose new teams from a limited group of current employees. This scenario is explored in other work [Lapp et al., 2019]. In the current work, all teams are composed by drawing from the virtual population. When plots compare agents or homogeneous teams of three styles, the KAI scores are 55 (adaptive), 95 (mid-range), and 135 (innovative). These represent the 1.5th, 50th, and 98.5th percentiles of the population; KAI $=55$ and $\mathrm{KAI}=135$ represent extremes of cognitive style behavior that are unlikely (but not impossible) to be observed in real life. These are intended to demonstrate the range of possible behaviors across the KAI spectrum. Organic composition gives more realistic team compositions.

\section{Objective functions}

The design problem is represented by a scalar objective function $f(\vec{x})$ of $n$ dimensions (variables). A solution defines the values of all variables, meaning a solution is a vector $\vec{x}$ in the n-dimensional space. The quality of the solution is defined as the objective function's value at that point, $f(\vec{x})$. The goal is to maximize solution quality. Real-world problems can sometimes be formulated as analytic objective functions with several variables and constraints, as in Zurita et al. [2017]. However, this paper implements a more abstract mathematical objective function, so that it can be tuned and scaled in predictable ways. The objective function used throughout this paper is a summation of a quadratic function and a sinusoidal function in the form:

$$
f(\vec{x})=\sum_{i=1}^{n} \alpha \cos \left(\frac{\omega \vec{x}_{i}}{\beta}\right)-C\left(\frac{\vec{x}_{i}}{\beta}\right)^{2} \quad \text { for }-0.5 \leq x_{i} \leq 0.5
$$


small amplitude $(\mathbf{a}=0.22)$

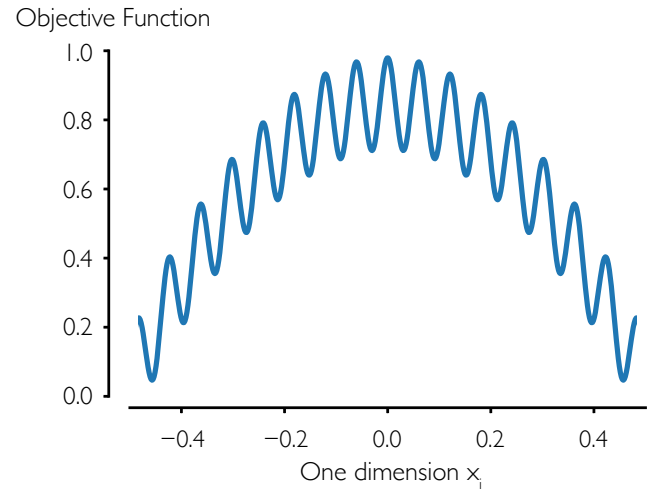

large amplitude $(\boldsymbol{a}=5)$

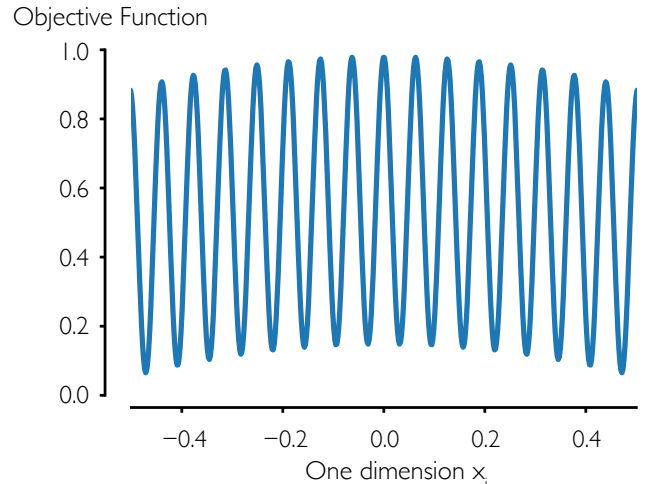

Figure 8. The objective function is a composite of a sinusoid and a parabola. The oscillation amplitude $\alpha$ of the objective function is varied from 0.22 to 5 . Global exploration of the space is important when $\alpha$ is small, but local exploitation is sufficient when $\alpha$ is large. The vertical axis shows normalized solution quality.

Figure 8 shows the objective function in one dimension for two values of $\alpha$. The objective function can be defined in any number of dimensions, $n$, and is symmetric in all dimensions. The oscillation frequency, $\omega$, and quadratic coefficient, $C$, are held constant. The space is bounded by $[-1,1]$ in all dimensions: solutions outside of this cube are infeasible. In order to focus on the relative performance of teams for a given problem, all performance axes for figures in this paper are normalized to the range of 0 to 1 .

This function is varied in two ways: (1) by scaling the independent variables in all dimensions using the scaling parameter, $\beta$, and; (2) by scaling the amplitude of the sinusoid, $\alpha$. The first parameter affects the size of the search space, while the second parameter affects the amplitude of the sinusoid. Changing these two characteristic values creates a variety of different problems that may favor different cognitive styles.

\section{Evaluating performance}

At the end of the simulation, each team's performance is the solution quality of the best solution any agent has had at any time during the simulation. Because the KABOOM model is stochastic, it repeats the same experiment 16 times (except where otherwise noted) before changing any parameters. However, due to the team selection methods described above, each repetition of the simulation uses a new team that is not composed of exactly the same agents. This is a standard Monte-Carlo simulation approach, in which random instantiations enable the exploration of team performance across a variety of compositions. In this paper, plots of results show the mean and standard deviation of team performance with a dot and vertical error bar (shown in blue unless there are multiple series on one plot). In some plots, separate team scores from each repetition are shown as gray dots. Linear and quadratic best-fit equations are displayed as red curves. 


\section{Computational performance of the model}

The Python implementation of KABOOM completes a simulation of 12 agents with 300 iterations and a 12-dimensional objective function in 4.1 seconds on a laptop with 16 gigabytes of RAM and a $2.5 \mathrm{GHz}$ Intel Core i7 Processor. The Python implementation of KABOOM is available on GitHub ${ }^{1}$.

\section{Results}

In this section, three studies examine how communication, specialization, and composition independently influence team performance for various cognitive style compositions. Communication refers to the frequency of pair-wise solution sharing and the frequency of team meetings. The first study shows that while pairwise communication can often help performance, the optimal communication rate depends strongly on the cognitive style of the team. Specialization refers to the division of the team into sub-teams which tackle pieces of the problem independently. Results from the specialization study suggest that the optimal amount of team specialization depends strongly on the cognitive styles of its members. Finally, twenty-five variations of the problem are created to test how differences in the problem affect the performance of agents with different cognitive styles. The performance of a team is measured by the objective function's value for the best solution any agent on the team has found through the course of the simulation.

\section{Communication: pairwise communication frequency}

Pairwise communication is driven by agents' desired communication frequency and has organic timing. Our first study examined how the frequency of pairwise communication affects performance. Results of previous work suggest that there is a curvilinear (rather than linear) relationship between communication and performance, meaning that the best performance will occur at some intermediate communication rate, above which performance will decrease [PatrashkovaVolzdoska et al., 2003, Patrashkova and McComb, 2004, McComb et al., 2017a].

We call the agents' rate of pairwise communication the "communication policy" c. At each iteration, every agent in the simulation chooses to collaborate with another agent with probability according to the communication policy $c$, or to explore individually with probability $1-c$. Varying $c$ from zero to one represents the spectrum of strategies from individual exploration with no pairwise communication $(c=0)$ to constant communication with no exploration $(c=1)$.

Figure 9A shows the performance of teams with organic composition (12 agents per team, 4 sub-teams of 3 ) for a range of communication policies. Each gray dot represents one team's final solution quality. The blue points and lines indicate the mean and one standard deviation for the eight teams' respective performance with a given communication policy. It is clear that there is a curvilinear relationship involved, where communication improves performance up to a point and then diminishes performance. (Performance drops significantly for $c=1$, because agents never explore the solution space.) The optimal policy

\footnotetext{
${ }^{1} \mathrm{KABOOM}$ is available at https://github.com/THREDgroup/kaboom/releases/tag/v1.0beta, and is licensed under the open source MIT license. Installation: pip install git+http://github.com/THREDgroup/kaboom@v1.0-beta
} 


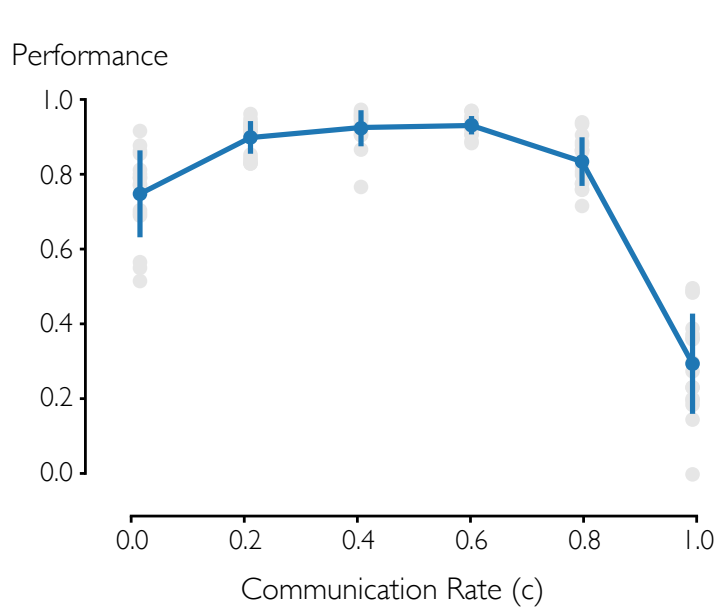

(A)

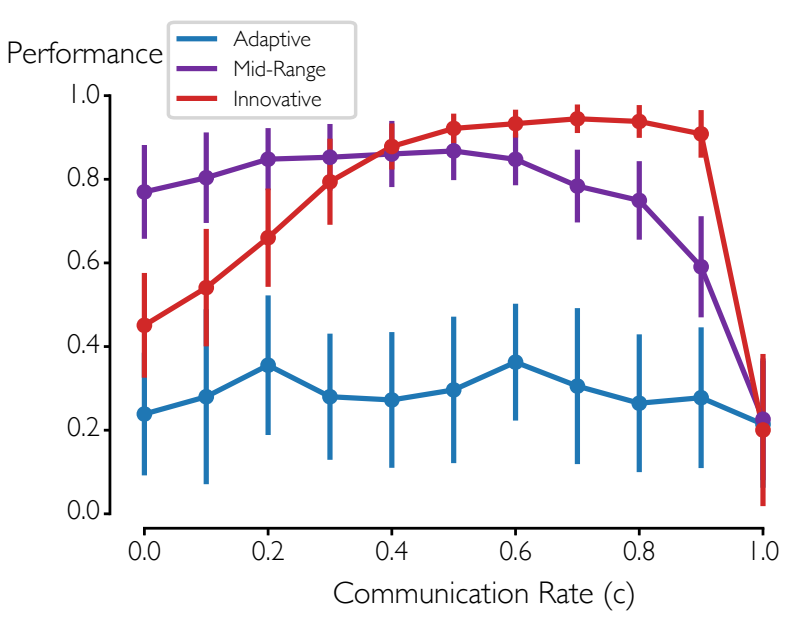

(B)

Figure 9. (A) Trade-off of pairwise communication frequency with team performance of 12-agent team with 4 subteams (B) Effect of communication frequency on team performance for homogeneous teams of adaptive, mid-range, and innovative style (in both plots, error bars indicate \pm 1 standard deviation

for this problem and team is to have a $40 \%$ to $60 \%$ chance of trying to collaborate on each turn. This result is consistent when testing with different team sizes and specializations.

The optimal communication policy changes drastically for homogeneous teams of different cognitive styles (Figure 9B). For a homogeneous team with a shared innovative style, increasing the communication rate increases performance up to very high values $(c=0.8)$. Compared to the effect on homogeneous innovative teams, the effect of communication is much weaker for the homogeneous adaptive teams, but there is a boost in performance at 0.2 and 0.6. The homogeneous mid-range team performs best for $c$ around 0.4 , similar to the organic teams.

The performance of homogeneous innovative teams with high rates of communication $(0.5<c<0.9)$ has a smaller standard deviation, as well as a higher average score. We can interpret this result by considering the balance of exploration (broad search) and exploitation (local refinement of a solution). An innovative team has little difficulty exploring the space, but they will not converge and refine the best solutions unless the team members interact very frequently, effectively "bringing themselves back" to the original problem-solving aim. For mid-range homogeneous teams, convergence and exploitation happen more easily, but too much time spent on pair-wise communication $(c>0.6)$ inhibits thorough exploration. For more adaptive homogeneous teams, performance is less dependent on communication frequency, as more adaptive teams tend to reach consensus early and then stick to the approach they have agreed upon, which tends to focus on refining the best solutions by default. These results agree well with a human-subjects study on Adaption-Innovation style and team network structures [Carnabuci and Dioszegi, 2015]. That study found that adaptors performed best in loosely connected networks with structural holes, while innovators performed best in densely connected networks. 
Table 2. Specialized teams decompose a problem by dividing the dimensions among sub-teams.

$\begin{array}{cccccccc}\text { Number of } & \text { Subteams } & x_{1} & x_{2} & x_{3} & x_{4} & x_{5} & x_{6} \\ \text { Subteams } & \text { A } & \text { A } & \text { A } & \text { A } & \text { A } & \text { A } & \text { A } \\ 1 & \text { A, B } & \text { A } & \text { A } & \text { A } & \text { B } & \text { B } & \text { B } \\ 2 & \text { A, B, C } & \text { A } & \text { A } & \text { B } & \text { B } & \text { C } & \text { C } \\ 3 & \text { A, B, C, D, E, F } & \text { A } & \text { B } & \text { C } & \text { D } & \text { E } & \text { F }\end{array}$

\section{Specialization: number of specialized subteams}

KABOOM can rapidly simulate team performance for many different team compositions and sub-team specialization configurations, giving insight into potential strategies for team organization. To demonstrate KABOOM's ability to study a wide range of scenarios, this section reports on the optimal amount of specialization in a team for different team sizes and style compositions.

Engineering teams often specialize by breaking a problem into (semi)independent pieces and assigning different parts of the problem to sub-teams [AustinBreneman et al., 2015, e.g.]. For example, a team designing a rocket might have sub-teams working on propulsion, stability, and aerodynamics. In the context of this research, specialization refers to the number of sub-teams working on independent aspects of a problem. The 'independent aspects of a problem' correspond to mutually exclusive sets of dimensions. This assumes a perfect decomposition of the variables of the problem, which is not always possible. However, this assumption allows KABOOM to study the effects of problem decomposition as a measure of team specialization. The agents on a team are evenly distributed across the sub-teams. For example, a team of six agents might be organized as one flat team of six agents, two sub-teams of three, three sub-teams of two, or six individuals all working on independent aspects of the problem. Each sub-team is specialized in that it controls a subset of all the dimensions in the problem. Table 2 illustrates how a 6-agent team would divide a 6-dimensional problem amongst sub-teams for different amounts of team specialization. Note that with regard to problem decomposition, six individuals working on six sub-teams (complete specialization) represents a distinct scenario from all six individuals working on 1 sub-team (no specialization).

To demonstrate the effect of specialization on performance, Figure 10 shows the optimal amount of specialization for 32-agent teams of organic composition. The bottom horizontal axis shows the number of agents per team, while the top horizontal axis shows the number of sub-teams. (Team size and number of agents per team determine the number of sub-teams.) The optimal specialization for 32agent teams or organic composition is sixteen sub-teams (pairs) of two agents. For cases in which the number of agents is not evenly divisible by the number of sub-teams, the sub-teams are not all equal in size. For instance, 32 agents cannot be equally divided into 10 sub-teams. In that case, the team was composed of 8 sub-teams of 3 agents and 2 sub-teams of 4 agents. Approximations of this nature are necessary for teams composed of 10,6, and 5 sub-teams.

The next investigation explores whether cognitive style affects the optimal 


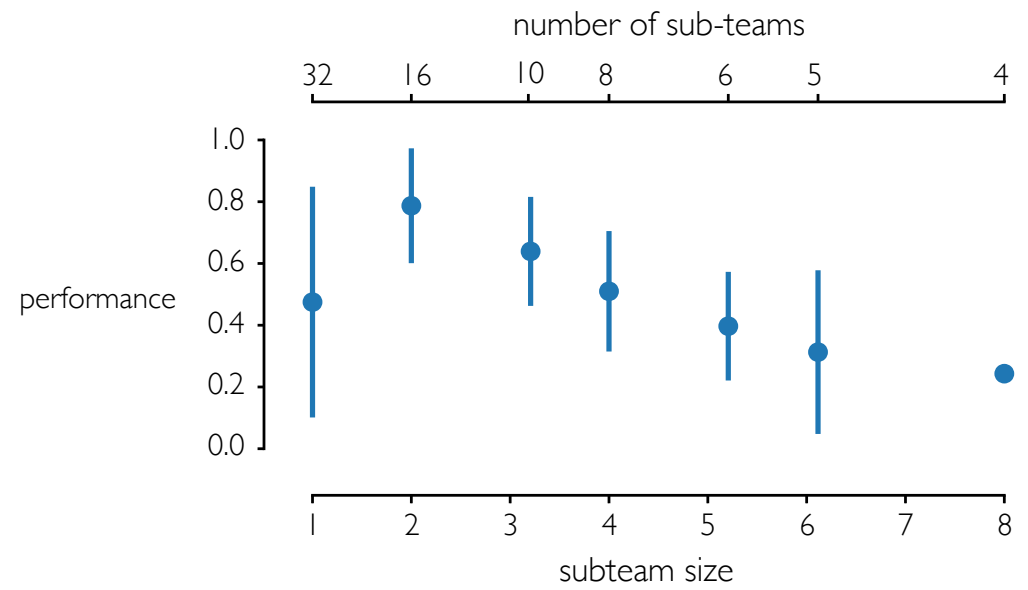

Figure 10. Performance of teams of 32 agents with organic composition, for different levels of team specialization (error bars indicate \pm 1 standard deviation). For cases in which the number of agents is not evenly divisible by the number of sub-teams, the subteams are not all equal in size.

amount of team specialization. We use the null hypothesis that the cognitive styles of agents on the team will not affect the optimal amount of specialization. Figure 11 shows the same study as above, but with homogeneous teams of more innovative, more adaptive, and mid-range styles rather than organically composed teams. The optimal specialization was significantly different for the three types of teams (Figure 11). The innovative teams perform best in larger sub-teams of four or eight agents, while the mid-range teams perform best with complete specialization (32 teams of one agent each), and the adaptive teams showed less dependence on specialization but perform best with three agents.

These results suggest that it may be important to consider the styles of team members when deciding how and how much to divide a team into specialized sub-teams. Specifically, the model predicts that a homogeneous team of more innovative individuals will perform poorly with small, highly specialized subteams. This result is consistent for problems with different values of $\alpha$ and $\beta$ (the objective function parameters). Complete specialization into 1-person sub-teams requires a high level of trust in each agent's performance, because every agent will contribute to the team's aggregate solution. However, innovative agents tend to perform less predictably (i.e., sometimes poorly and sometimes well), making it easier for smaller innovative sub-teams to go off track. Having larger teams of four to eight innovators allows the full team to take the best solution of one agent on each sub-team and discard lower quality solutions.

Mid-range teams, on the other hand, perform best with complete specialization into one-person sub-teams. These agents do not explore radical solutions in potentially damaging ways, but they do explore the space sufficiently to find good solutions. This trend reflects the results of Blackburn et al. [2006], a study of software development teams which concluded that increasing team size decreased productivity. Finally, homogeneous adaptive teams do not explore radical solutions that could result in poor performance, but they also do not explore the solution space sufficiently to find high-quality solutions. The effect 


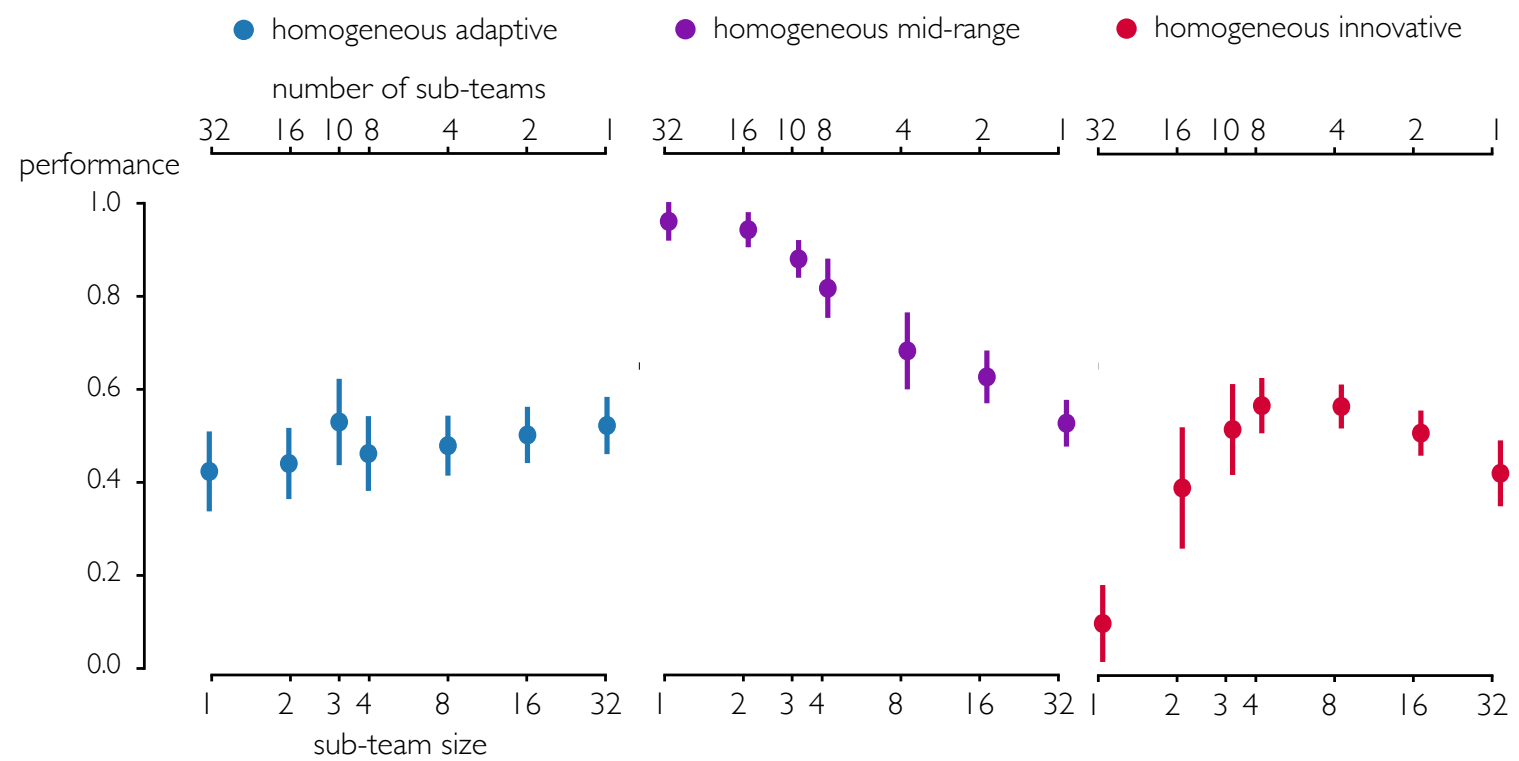

Figure 11. Performance versus specialization for three homogeneous teams of different KAI cognitive styles (error bars indicate \pm 1 standard deviation). For cases in which the number of agents is not evenly divisible by the number of sub-teams, the sub-teams are not all equal in size.

of team specialization on their performance is small, which is likely a result (once again) of the high degree of consistency found within adaptive teams due to their preference for consensual decision-making. In future work, human subjects studies will be needed to test the hypotheses suggested by all of these results i.e., for more adaptive, more innovative, and mid-range teams of various amounts of specialization.

\section{Composition: performance of homogeneous style teams on diverse problems}

This section analyzes the performance of homogeneous teams of different shared KAI total scores on a matrix of 25 problems. These problems were created by modifying the objective function parameters - i.e., by permuting five logarithmically spaced values for $\alpha$ and five logarithmically spaced values for $\beta$.

The first parameter $(\alpha)$ affects the amplitude of the sinusoid (Figure 8 ). When the amplitude is large relative to the quadratic function, the quadratic becomes negligible, so that optimizing any local minimum yields similar performance to finding the global minimum. On the other hand, when the amplitude is small, the quadratic function becomes important in the cost function, so that finding the global local minimum is much better than finding a distant local minimum. Because large amplitude problems reward local exploration, while small amplitude problems reward global exploration, we hypothesize that more adaptive agents (lower KAI scores) will outperform other styles on large-amplitude problems (large $\alpha$ ), while more innovative agents (higher KAI scores) will have an advantage in small-amplitude (small $\alpha$ ) problems. The second parameter $(\beta)$ affects the size of the search space. Large search spaces 


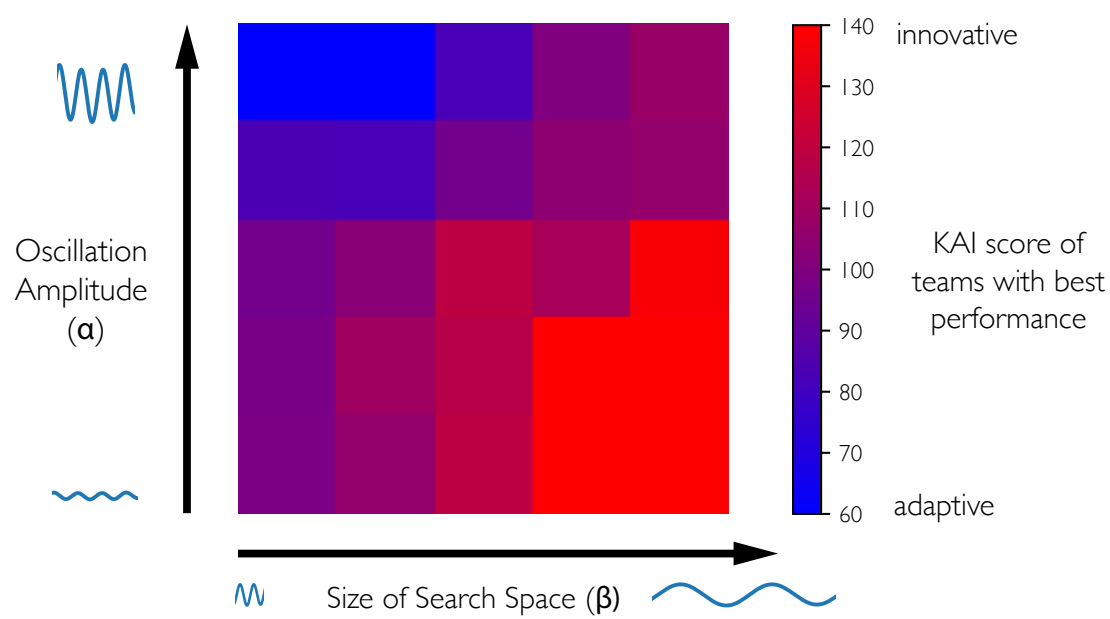

Figure 12. Homogeneous teams of different KAI styles were tested on each of 25 problems, with varying $\alpha$ and $\beta$. Color indicates the best-performing style for each problem from blue (more adaptive) to red (more innovative).

require broader search strategies and more stochastic methods, corresponding to higher temperature in a simulated-annealing paradigm [Kirkpatrick et al., 1983]. Therefore, we hypothesize that higher (more innovative) KAI scores will be advantageous for large search spaces (large $\beta$ ), while lower (more adaptive) KAI scores will have an advantage in small search spaces (small $\beta$ ), with mid-range KAI scores having an advantage in between.

Homogeneous teams of more adaptive, more innovative, and mid-range styles, respectively, were tested on the 25 problems. Each combination of $\alpha$, $\beta$, and shared KAI score was repeated only 8 times due to the large number of combinations. Figure 12 shows the optimal style of a homogeneous team on the KAI spectrum for each problem (from red as the most innovative homogeneous team to blue as the most adaptive homogeneous team). The optimal style of the problems ranges from highly innovative in the upper-left corner (large search space, small sinusoidal amplitude), to mid-range, to highly adaptive in the lowerright corner (small search space, large sinusoidal amplitude).

Both our hypotheses about the alignment of shared team cognitive style and problem characteristics proved to be correct: larger search spaces favor innovators, and larger sinusoidal amplitudes favor adaptors. The combined effect of the two problem variables is that extreme innovators perform best for problems with small $\alpha$ and large $\beta$ (lower right corner of the figure), extreme adaptors perform best for problems with large $\alpha$ and small $\beta$ (upper left), and mid-range styles perform best on other combinations of $\alpha$ and $\beta$. It is worth noting that there are some problems where having a mid-range style is advantageous over a more adaptive or more innovative style, which validates the importance of remembering that cognitive style is continuous, rather than a dichotomy of two "types" [Kirton, 2003].

The $\alpha$ and $\beta$ parameters of the simulated problems are loosely related to the characteristics of real-life problems that make them better suited for adaptive or 
innovative approaches. The oscillation amplitude $\alpha$ aligns with the importance of global exploration versus local exploitation. Real-world design problems focused on new product development generally require broad exploration (as in designing a new children's toy), while redesigns and improvements of existing designs require thorough local exploration (as in improving the efficiency of an internal combustion engine). The effective solution space size $\beta$ corresponds to the number of iterations required to explore a space given a constant step size. Real-world problems may have small solution spaces when there are few variables, when variables only take a limited number of discrete values, or when there are a small number of distinguishable solutions. Conversely, problems can have large solution spaces when they have many dimensions or continuous variables with many distinguishable values. For example, a traveling salesman problem [Applegate et al., 2007] may have a small solution space if it only has a few nodes, but a very large solution space if it has many nodes. Future work will incorporate contextualized design problems in the model to better understand the links between computational objective functions and real world problems.

\section{Limitations}

The KABOOM model focuses on specific characteristics of cognitive style and team problem solving; it does not create a comprehensive representation of team problem solving. Because of this, there are several major limitations of the current model. With regard to cognitive style, the model attempts to map specific human problem-solving characteristics to parameters in the model (i.e. mapping the diversity of people's solutions to the step size and temperature in a simulated annealing framework). Cognitive style and behavior are extremely complex, and many important effects of style have not been modeled. For instance, KABOOM does not attempt to model the coping behaviors that people of different styles may use when working together. Because modeling all aspects of human behavior would be unfeasible, we were forced to choose which aspects of behavior were most important to team performance outcomes and to envision how they would map onto quantitative parameters in the model.

The model's reflection of communication, collaboration, and specialization are also limited. Previous work has focused extensively on modeling communication and collaboration within teams [Fan and Yen, 2004, Singh et al., 2013, Patrashkova-Volzdoska et al., 2003, Patrashkova and McComb, 2004, Bernstein et al., 2018, Tsvetovat and Carley, 2004], resulting in models with much more complex mechanisms for communication than those in KABOOM. In real teams, people communicate ideas about process, strategy, sentiment, and emotion, while agents in KABOOM only communicate their solutions. While more complex models of communication could be incorporated into KABOOM, the mechanisms implemented for pair-wise solution sharing and team convergence with meetings were sufficient to recreate the communication trade-offs observed in the literature. Likewise, previous work has developed more complex constructs for team specialization that incorporate communication patterns, domain knowledge, and heterogeneous cognitive abilities [Fan and Yen, 2004, Hulse et al., 2017, 2018]. In KABOOM, specialization is treated simply as a problem decomposition, which may be an over-simplification of how organizational structure shapes team performance.

Further, this work only tests KABOOM with one parameterized design 
problem that is abstract and hard to relate to real-world problems. The model has the capacity to study any design problem that can be posed as an objective function, but additional problems were beyond the scope of this paper. Because the experiments from this paper are all based on a set of closely-related objective functions that does not model a real-world problem, their results cannot be directly compared to real-world design teams or solutions. Future studies will incorporate contextualized real-world design problems into KABOOM to provide more direct comparison to real-world scenarios.

The lack of any human subjects research to test the validity of results from the model is another important limitation of the current work. Without support from human-subjects validation studies, the results presented in this paper cannot be assumed to hold true in real-life scenarios. Future work will focus on validating model results with human-subjects studies. All of these limitations are a reminder that this model is an early experiment in the direction of simulating human cognitive style with agent-based modeling. The results from this new model are as much demonstration of the method's capabilities for studying teamwork as they are predictions of real-world behavior. The limitations noted here also lay the groundwork for the future work described below.

\section{Future work}

This section first addresses future work specific to KABOOM and then more broadly outlines future work for the field of design science. First, future work on KABOOM should focus on (1) validating the results of the agent-based model with human-subjects research; (2) enhancing the cognitive and social complexity of the agents to more closely reflect human activity; and (3) mapping between real-world and mathematically defined problems. Results from the model have the potential to inform the design of focused case studies that test specific hypotheses about team behavior and performance. For instance, a humansubjects study could test the hypothesis that homogeneous teams of innovators will perform best with very frequent communication, while homogeneous teams of mid-range agents will perform best with less communication (as in Figure 9B). Discrepancies between real-world behavior and model behavior can then inform the refinement of the model through tuning of parameters, addition of higher-level cognitive processes, and adjustment of the fundamental behavioral rules used in the model.

KABOOM could also be expanded to include more complex behaviors, as seen in other contemporary models. For instance, the model could adopt social networking patterns as in Singh et al. [2013]. Moreover, coping behavior the temporary adjustments in behavior that people make to bridge large cognitive style gaps could be incorporated into agents' behavior. The agents could also be given learning strategies such as sequence-learning, as in McComb et al. [2017b]. These modifications could result in a more nuanced model of team performance and behavior with greater predictive power.

Another important direction of future work is to establish an approach to map between the mathematically-defined problems commonly used in a simulation and real-world problems that are often messy and ill-defined. Using an objective function that is contextualized within a realistic problem can allow simulation results to be compared more easily to human-subjects tests on the same problem. For example, McComb et al. [2015] directly compares trusses 
designed by humans in a cognitive study and virtual agents in the CISAT simulation. However, real-world design problems are generally ill-defined, and solutions often cannot be represented by a set of quantitative design variables. These ill-defined problems might be simulated by imposing the mathematical structure seen in simulated problems, but this inherently locks the problem into a single representation. Therefore, future work will need to develop a method of mapping between simulated problems and real-world problems without imposing a strictly-defined mathematical structure. This is critical for transferring the results of computational simulations into real-world practice.

More broadly, a better understanding of design teams can be achieved through a variety of future research directions in the field of design science. First, simulations should strive to model individual differences between people, rather than assuming people are homogeneous. These differences are not limited to cognitive style and could include individual traits such as learning ability, background knowledge, social connectedness, or personality. Next, future work will need to explore the relationships between these individual traits and attributes of a design problem. These relationships will help to predict an individual's performance on a specific problem, which could be used for determining team composition.

One of the main attractions of simulation is the ability to rapidly explore large numbers of hypothetical scenarios at relatively low cost. This strength should be leveraged in research to explore many scenarios in simulation before investing in human subjects experimentation. Through this hybrid approach, modeling can act as an early-prototyping phase, where many scenarios are considered and those with the greatest potential for knowledge creation are selected for human subjects studies. However, the benefits of this approach may extend far beyond academia. Given high-performance simulation models of design teams, many industries will be able to incorporate simulation into their human resources and management practices. By simulating thousands of teams before actually assembling one, organizations will be able to create more successful teams more reliably, leading to significant savings in cost and increases in productivity.

\section{Conclusion}

This paper introduced $\mathrm{KABOOM}$, the first team simulation modeling framework to reflect the diverse range of human cognitive styles. In KABOOM, the behaviors of heterogeneous agents simulate the range of cognitive styles described by the Kirton Adaption-Innovation spectrum. The primary objectives of this research were to create an agent-based model incorporating cognitive style, analyze the effects of style on problem-solving performance, and explore the interactions of style with team composition, specialization, and communication. The development of cognitive style in the simulated agents took inspiration from research in psychology, sociology, design science, and Kirton's AdaptionInnovation theory to reflect empirically observed human behaviors. The resulting model successfully simulated a range of distinct behaviors driven by cognitive style. In KABOOM simulations, the behavior of more adaptive, mid-range, and more innovative agents generally reflected the intended behavior and resulted in patterns in performance that were sometimes non-intuitive. The key hypotheses dervied from the computational experiments are as follows: 
1. The optimal communication rate for innovative teams is higher than that of adaptive and mid-range teams.

2. Extreme specialization benefits mid-range teams but can be harmful for innovative teams.

3. The optimal cognitive style for a problem depends on the size of the solution space and importance of global exploration.

While this work has focused on developing a flexible simulation framework, future work will validate and further develop the model through a targeted set of human studies. The most powerful models and insights are likely to result from a hybrid research approach that merges the breadth and flexibility of computational simulation with the validation and holistic reality of human studies. The human studies will not only test the validity of insights provided by the model, but will also serve as a feedback loop for tuning and improving the behavior of agents in the model. Likewise, insights from the model will guide the development of human studies designed to examine specific hypotheses about behavior, teamwork, and cognitive style, which could lead to novel insights on social and cognitive behaviors. In time, the mutual feedback between in vivo and in silico scientific investigations will lead to a deeper understanding of the cognitive processes underlying design, and how those processes influence the behaviors of individuals and teams. By allowing computational agents to embody unique cognitive styles, this model opens the door to a future of design analysis built at the intersection of computer precision and human individuality.

\section{Acknowledgements}

This research was supported with funding from the School of Engineering Design, Technology, and Professional Programs at The Pennsylvania State University.

\section{References}

Richard J Riding. On the Nature of Cognitive Style. Educational Psychology, 17:29-49, 1997. ISSN 1469-5820. doi: 10.1080/0144341970170102.

M. J. Kirton. Adaption-innovation: In the context of diversity and change. 2003. ISBN 0203695003. doi: 10.4324/9780203695005.

Kathryn Jablokow. Developing Problem Solving Leadership: A Cognitive Approach. International Journal of Engineering Education, 24(5):936-954, 2008. ISSN 0949149X.

Kathryn W. Jablokow, Wesley Teerlink, Seda Yilmaz, Shanna R. Daly, and Eli M. Silk. The impact of teaming and cognitive style on student perceptions of design ideation outcomes. ASEE Annual Conference and Exposition, Conference Proceedings, 122nd ASEE(122nd ASEE Annual Conference and Exposition: Making Value for Society), 2015a. ISSN 21535965.

Neeraj Sonalkar, Kathryn Jablokow, Jonathan Edelman, Ade Mabogunje, and Larry Leifer. Design Whodunit: The Relationship Between Individual Characteristics and Interaction Behaviors in Design Concept Generation. In Volume 7: 29th International Conference on Design Theory and Methodology, page V007T06A009. ASME, aug 2017. ISBN 978-0-7918-5821-9. doi: 10.1115/DETC2017-68239. 
Michael Kirton. Adaptors and innovators: A description and measure. Journal of Applied Psychology, 61(5):622-629, 1976. ISSN 00219010. doi: 10.1037/0021-9010.61.5.622.

Kathryn W. Jablokow and David E. Booth. The impact and management of cognitive gap in high performance product development organizations. Journal of Engineering and Technology Management - JET-M, 23(4):313-336, 2006. ISSN 09234748. doi: 10.1016/j.jengtecman.2006.08.003.

Kathryn W. Jablokow, Joanna F. Defranco, Sally Sue Richmond, Michael J. Piovoso, and Sven G. Bilén. Cognitive Style and Concept Mapping Performance. Journal of Engineering Education, 104(3):303-325, 2015b. ISSN 10694730. doi: 10.1002/jee.20076.

Raymond E Levitt. The Virtual Design Team: Designing Project Organizations as Engineers Design Bridges. Journal of Organization Design, 1(2):14, 2012. ISSN 2245-408X. doi: 10.7146/jod.6345.

Richard M. Crowder, Mark A. Robinson, Helen P.N. Hughes, and Yee Wai Sim. The development of an agent-based modeling framework for simulating engineering team work. IEEE Transactions on Systems, Man, and Cybernetics Part A:Systems and Humans, 42(6):1425-1439, 2012. ISSN 10834427. doi: 10.1109/TSMCA.2012.2199304.

Yoav Bergner, Jessica J Andrews, Mengxiao Zhu, and Joseph E Gonzales. Agent-Based Modeling of Collaborative Problem Solving. ETS Research Report Series, 2 (December), 2016.

MM Perisic, Tomislav Martinec, M Storga, T Kanduc, Marija Majda Perišić, Tomislav Martinec, Mario Štorga, and Tadej Kanduč. Agent-Based Simulation Framework To Support Management of Teams Performing Development Activities. Design2016, pages 1925-1936, 2016.

Vishal Singh, Andy Dong, and John S. Gero. Social learning in design teams: The importance of direct and indirect communications. Artificial Intelligence for Engineering Design, Analysis and Manufacturing: AIEDAM, 27(2):167-182, 2013. ISSN 08900604. doi: 10.1017/S0890060413000061.

Xiaocong Fan and John Yen. Modeling and simulating human teamwork behaviors using intelligent agents. Physics of Life Reviews, 1(3):173-201, dec 2004. ISSN 1571-0645. doi: 10.1016/J.PLREV.2004.10.001.

Daniel Hulse, Brandon Gigous, Kagan Tumer, Christopher Hoyle, and Irem Y Tumer. Towards a Distributed Multiagent Learning-Based Design Optimization Method. In Volume 2A: 43rd Design Automation Conference, page V02AT03A008, 2017. ISBN 978-0-7918-5812-7. doi: 10.1115/DETC2017-68042.

Christopher McComb, Jonathan Cagan, and Kenneth Kotovsky. Lifting the Veil: Drawing insights about design teams from a cognitively-inspired computational model. Design Studies, 40:119-142, 2015. ISSN 0142-694X. doi: 10.1016/j.destud.2015.06.005.

Maksim Tsvetovat and Kathleen M. Carley. Modeling Complex Socio-technical Systems using Multi-Agent Simulation Methods. KI, pages 23-28, 2004.

NFS Zurita, MK Colby, Irem Tumer, Christopher Hoyle, and Kagan Tumer. Design of Complex Engineered Systems Using Multi-Agent Coordination. Journal of Computing and Information Science in Engineering, 18(October):1-13, 2017. ISSN 1530-9827. doi: 10.1115/1.4038158.

Terri R. Kurtzberg. Feeling creative, being creative: An empirical study of diversity and creativity in teams. Creativity Research Journal, 2005. ISSN 10400419. doi: 10.1207/s15326934crj1701_5.

Peter K Hammerschmidt. The Kirton Adaption Innovation Inventory and Group Problem Solving Success Rates, 1996. 
Rosanna Garcia. Uses of agent-based modeling in innovation/new product development research. Journal of Product Innovation Management, 22(5):380-398, 2005. ISSN 07376782. doi: 10.1111/j.1540-5885.2005.00136.x.

Eric Bonabeau. Agent-based modeling: methods and techniques for simulating human systems. Proceedings of the National Academy of Sciences, 99(suppl. 3):7280-7287, 2002. ISSN 0027-8424. doi: 10.1073/pnas.082080899.

Nicholas R. Jennings. On agent-based software engineering. Artificial Intelligence, 117 (2):277-296, mar 2000. ISSN 0004-3702. doi: 10.1016/S0004-3702(99)00107-1.

Ralitza R. Patrashkova-Volzdoska, Sara A. McComb, Stephen G. Green, and W. Dale Compton. Examining a curvilinear relationship between communication frequency and team performance in cross-functional project teams. IEEE Transactions on Engineering Management, 50(3):262-269, 2003. ISSN 00189391. doi: 10.1109/TEM.2003.817298.

Ralitza R Patrashkova and Sara A McComb. Exploring why more communication is not better: insights from a computational model of cross-functional teams. J. Eng. Technol. Manage, 21:83-114, 2004. doi: 10.1016/j.jengtecman.2003.12.005.

Ethan Bernstein, Jesse Shore, and David Lazer. How intermittent breaks in interaction improve collective intelligence. Proceedings of the National Academy of Sciences, 115(35):201802407, 2018. ISSN 0027-8424. doi: 10.1073/pnas.1802407115.

Christopher McComb, Jonathan Cagan, and Kenneth Kotovsky. Optimizing Design Teams Based on Problem Properties: Computational Team Simulations and an Applied Empirical Test. Journal of Mechanical Design, 139(4):041101, 2017a. ISSN 1050-0472. doi: 10.1115/1.4035793.

Christopher McComb and Torsten Maier. Designing Improved Teams for Crowdsourced Competitions. In Volume 7: 30th International Conference on Design Theory and Methodology, page V007T06A025. ASME, aug 2018. ISBN 978-0-7918-5184-5. doi: 10.1115/DETC2018-85457.

Daniel Hulse, Kagan Tumer, Christopher Hoyle, and Irem Tumer. Modeling multidisciplinary design with multiagent learning. Artificial Intelligence for Engineering Design, Analysis and Manufacturing: AIEDAM, 2018. ISSN 14691760. doi: $10.1017 /$ S0890060418000161.

Juan Martínez-Miranda and Juan Pavón. Modeling the influence of trust on work team performance. Simulation, 88(4):408-436, 2012. ISSN 17413133. doi: $10.1177 / 0037549711404714$.

Xiaodong Zhang, Le Luo, Yu Yang, Yingzi Li, Christopher M Schlick, and Morten Grandt. A simulation approach for evaluation and improvement of organisational planning in collaborative product development projects. International Journal of Production Research, 47(13):3471-3501, 2009. ISSN 0020-7543. doi: 10.1080/00207540802356770.

Shona L Brown and Kathleen M Eisenhardt. PRODUCT DEVELOPMENT: PAST RESEARCH, PRESENT FINDINGS, AND FUTURE DIRECTIONS. Technical Report 2, 1995.

John C Kunz, Tore R Christiansen, Geoff P Cohen, Yan Jin, and Raymond E Levitt. A computational simulation model of project organizations that is usable and predictive for routine, project-oriented design tasks. Technical Report 11, 1998.

João Ventura Fernandes, Elsa Henriques, Arlindo Silva, and César Pimentel. Modelling the dynamics of complex early design processes: an agent-based approach. Design Science, 3:e19, 2017. ISSN 2053-4701. doi: 10.1017/dsj.2017.17.

Sean D Vermillion and Richard J Malak. Using a principal-agent model to investigate delegation in systems engineering. In ASME 2015 International Design Engineering Technical Conferences and Computers and Information in Engineering Conference, 
pages V01BT02A046-V01BT02A046. American Society of Mechanical Engineers, 2015. doi: 10.1115/DETC2015-47778.

Sean D Vermillion and Richard J Malak. A theoretical look at the impact of incentives on design problem effort provision. In ASME 2018 International Design Engineering Technical Conferences and Computers and Information in Engineering Conference, pages V007T06A030-V007T06A030. American Society of Mechanical Engineers, 2018. doi: 10.1115/DETC2018-85845.

J Martinez-Miranda, A Aldea, R Bañares-Alcantara, and M Alvarado. TEAKS: Simulation of Human Performance at Work to Support Team Configuration. Technical report, 2006.

Farnaz Motamediyan Dehkordi, Anthony Thompson, and Tobias Larsson. Impacts of Project-Overload on Innovation inside Organizations: Agent-Based Modeling. International Journal of Social, Behavioral, Educational, Economic, Business and Industrial Engineering, 6(11):2808-2813, 2012.

Christoph Urban and Bernd Schmidt. PECS-Agent-Based Modelling of Human Behaviour. Technical report, 2001.

Christopher McComb, Jonathan Cagan, and Kenneth Kotovsky. Capturing Human Sequence-Learning Abilities in Configuration Design Tasks Through Markov Chains. Journal of Mechanical Design, 139(9):091101, 2017b. ISSN 1050-0472. doi: 10.1115/1.4037185.

S Kirkpatrick, C D Gelatt, and M P Vecchi. Optimization by simulated annealing. Science (New York, N.Y.), 220(4598):671-80, may 1983. ISSN 0036-8075. doi: 10.1126/science.220.4598.671.

Jonathan Cagan and Kenneth Kotovsky. Simulated Annealing and the Generation of the Objective Function: A Model of Learning During Problem Solving. Computational Intelligence, 13(4):534-581, nov 1997. ISSN 0824-7935. doi: 10.1111/0824-7935.00051.

Bo Yang Yu, Tomonori Honda, Mostafa Sharqawy, and Maria Yang. Human behavior and domain knowledge in parameter design of complex systems. Design Studies, 45: 242-267, jul 2016. ISSN 0142694X. doi: 10.1016/j.destud.2016.04.005.

Andrew M Colman. A dictionary of psychology. Oxford University Press, USA, 2015.

Samuel Lapp, Kathryn Jablokow, and Christopher McComb. Collaborating with style: Using an agent-based model to simulate cognitive style diversity in problem solving teams. In ASME 2019 International Design Engineering Technical Conferences and Computers and Information in Engineering Conference. American Society of Mechanical Engineers, 2019. doi: 10.1115/DETC2019-97932.

Gianluca Carnabuci and Balint Dioszegi. Social Networks, Cognitive Style, and Innovative Performance: a Contingency Perspective. Academy of Management Journal, 58(3):881-905, 2015. ISSN 0001-4273. doi: 10.5465/amj.2013.1042.

Jesse Austin-Breneman, Bo Yang Yu, and Maria C. Yang. Biased Information Passing Between Subsystems Over Time in Complex System Design. Journal of Mechanical Design, 138(1):011101, nov 2015. ISSN 1050-0472. doi: 10.1115/1.4031745.

Joseph Blackburn, Michael A. Lapre, and Luk N Van Wassenhove. Brooks' Law Revisited: Improving Software Productivity by Managing Complexity. 2006.

David L Applegate, Robert E Bixby, and Vasek Chvátal. The Traveling Salesman Problem. Princeton University Press, 2007. 


\section{Parameters Table}

Table 3 lists the parameters used for each figure in the paper. The value var indicates that the parameter was an independent variable in the figure. Style Composition is indicated by $\mathrm{h}$ (homogeneous) or o (organic). Except when noted explicitly in the figure, homogeneous teams had a KAI score of 95 (the observed mean).

Table 3. Model parameters for all figures

\begin{tabular}{lccccc}
\hline Parameter & \multicolumn{5}{c}{ Value in Figures: } \\
\hline & $10 \mathrm{~A}$ & $10 \mathrm{~B}$ & 11 & 12 & 13 \\
\hline Team & & & & & \\
\hline Team Size & 12 & 12 & 32 & 32 & 6 \\
Number of Sub-Teams & 4 & 4 & var & var & 2 \\
Agents per sub-team & 3 & 3 & var & var & 3 \\
Style Composition & $\mathrm{h}$ & $\mathrm{h}$ & $\mathrm{o}$ & $\mathrm{h}$ & $\mathrm{h}$ \\
\hline Agents & & & & & \\
\hline Avg. Start Temp & 1 & 1 & 1 & 1 & 1 \\
Stdev. Temp & 0.8 & 0.8 & 0.8 & 0.8 & 0.8 \\
Avg. Start Speed & .07 & .07 & 0.01 & 0.01 & var \\
Stdev. Speed & .007 & .007 & .007 & .007 & var \\
\hline Communication & & & & & \\
\hline Comm Frequency & var & var & 0.2 & 0.2 & 0.2 \\
Meeting Interval & 50 & 50 & 50 & 50 & 50 \\
\hline Objective Function & & & & & \\
\hline Dimensions & 12 & 12 & 32 & 32 & 10 \\
Oscillation amplitude & 0.025 & 0.025 & 1 & 1 & var \\
Scaling parameter & 1 & 1 & 1 & 1 & var \\
Iterations & 300 & 300 & 300 & 300 & 300 \\
Experiment Repetitions & 16 & 32 & 16 & 16 & 8 \\
\hline & & & & &
\end{tabular}

